\title{
NON-REDUCED MODULI SPACES OF SHEAVES ON MULTIPLE CURVES
}

\author{
JEAN-MARC DRÉZET
}

\begin{abstract}
RESUME. Some coherent sheaves on projective varieties have a non reduced versal deformation space. For example, this is the case for most unstable rank 2 vector bundles on $\mathbb{P}_{2}$ (cf. [18]). In particular, it may happen that some moduli spaces of stable sheaves are non reduced.

We consider the case of some sheaves on ribbons (double structures on smooth projective curves): the quasi locally free sheaves of rigid type. Le $E$ be such a sheaf.

- Let $\mathcal{E}$ be a flat family of sheaves containing $E$. We find that it is a reduced deformation of $E$ when some canonical family associated to $\mathcal{E}$ is also flat.

- We consider a deformation of the ribbon to reduced projective curves with two components, and find that $E$ can be deformed in two distinct ways to sheaves on the reduced curves. In particular some components $\mathbf{M}$ of the moduli spaces of stable sheaves deform to two components of the moduli spaces of sheaves on the reduced curves, and $\mathbf{M}$ appears as the "limit" of varieties with two components, whence the non reduced structure of $\mathbf{M}$.
\end{abstract}

\section{SUMMARY}

1. Introduction

2. Preliminaries 6

3. Quasi locally free sheaves of rigid type 10

4. Coherent sheaves on reducible deformations of primitive double curves 13

5. Kodaïra-Spencer elements 16

References

Mathematics Subject Classification : 14D20, 14B20

\section{INTRODUCTION}

Let $X$ be a projective variety over $\mathbb{C}$ and $E$ a coherent sheaf on $X$. Let $\left(V_{E}, v_{0}, \mathcal{E}_{E}, \alpha\right)$ be a semi-universal deformation of $E\left(\left(V_{E}, v_{0}\right)\right.$ is a the germ of an analytic variety, $\mathcal{E}_{E}$ is a coherent sheaf on $X \times V_{E}$ and $\alpha: \mathcal{E}_{E, s_{0}} \rightarrow E$ is an isomorphism, cf. [17]). It may happen that $V_{E}$ is not reduced at $s_{0}$, for example in the case of unstable rank-2 vector bundles on $\mathbb{P}_{2}$ (cf. 1.2), or for some sheaves on ribbons. It is then natural to ask why $V_{E}$ is not reduced.

Notation: In this paper, an algebraic variety is a quasi-projective scheme over $\mathbb{C}$. 


\subsection{NON-REDUCED DEFORMATIONS OF SHEAVES}

1.1.1. The general case - If $\mathcal{F}$ is a flat family of sheaves parametrised by the germ $\left(T, t_{0}\right)$ of an analytic variety, and if $\mathcal{F}_{t_{0}} \simeq E$, there is a morphism $\phi:\left(T, t_{0}\right) \rightarrow\left(V_{E}, v_{0}\right)$ such that $\left(I_{X} \times \phi\right)^{*}\left(\mathcal{E}_{E}\right) \simeq \mathcal{F}$, with a uniquely defined tangent map

$$
T_{t_{0}} T \longrightarrow T_{v_{0}} V_{E}=\operatorname{Ext}_{\mathcal{O}_{X}}^{1}(E, E) \text {. }
$$

If $T$ is reduced then $\phi$ can be factorized to $V_{E, r e g}$, the reduced germ associated to $V_{E}$ :

$$
\phi: T \stackrel{\phi^{\prime}}{\longrightarrow} V_{E, \text { reg }} \longrightarrow V_{E} .
$$

It is then natural to ask:

(i) What is the tangent space $T_{v_{0}} V_{E, r e g} \subset \operatorname{Ext}_{\mathcal{O}_{X}}^{1}(E, E)$ ?

(ii) Under which conditions on $\mathcal{F}$, when $T$ is non reduced, is $\phi$ a morphism to $V_{E \text {,reg }}$ ?

and the more vague question

(iii) Why can $V_{E}$ be non reduced?

The problem can also be stated in terms of moduli spaces of stable sheaves: let $\mathcal{O}_{X}(1)$ be an ample line bundle on $X$ and $P_{E}$ the Hilbert polynomial of $E$. Let $\mathbf{M}$ be the moduli space of sheaves on $X$, stable with respect to $\mathcal{O}_{X}(1)$, and with Hilbert polynomial $P_{E}$. Suppose now that $E$ is stable. We can then ask

(i') What is the tangent space $T_{E} \mathbf{M}_{r e g} \subset T_{E} \mathbf{M}$ ?

(ii') If $\mathcal{F}$ is a flat family of stable sheaves of Hilbert polynomial $P_{E}$, parametrised by a variety $T$, we get a morphism $\phi: T \rightarrow \mathbf{M}$. Under which conditions on $\mathcal{F}$, when $T$ is non reduced, is $\phi$ a morphism to $\mathbf{M}_{r e g}$ ?

(iii') Why can $\mathbf{M}$ be non reduced?

For questions (ii), in the case of unstable rank-2 vector bundles on $\mathbb{P}_{2}$ and of some sheaves on multiple curves studied here, it appears that the bundles (or sheaves) have a canonical extra structure, and that $\phi$ is a morphism to $V_{E, r e g}$ when this structure is also flat over $V_{E}$. The case unstable rank- 2 vector bundles on $\mathbb{P}_{2}$ is briefly recalled in 1.2 .

1.1.2. The case of sheaves on ribbons - Good families of sheaves - In this paper we will investigate the case of some sheaves on ribbons (i.e double structures on smooth projective curves). Let $Y$ be a ribbon and $C=Y_{\text {reg }}$. For vector bundles $V_{E}$ is smooth (because in this case $\left.\operatorname{Ext}_{\mathcal{O}_{Y}}^{2}(E, E)=\{0\}\right)$. We will consider quasi-locally free sheaves of rigid type (defined and studied in [6]), i.e. coherent sheaves locally isomorphic to $a \mathcal{O}_{Y} \oplus \mathcal{O}_{C}$ for some integer $a$. Deformations of these sheaves are also quasi-locally free sheaves of rigid type, and $\operatorname{deg}\left(E_{\mid C}\right)$ is invariant by deformation.

For these sheaves, for (i) we have from [6]

$$
T_{v_{0}} V_{E, r e g}=H^{1}(\operatorname{End}(E)) .
$$

Let $L$ be the ideal sheaf of $C$ in $Y$. It is a line bundle on $C$. We will prove that

$$
T_{v_{0}} V_{E} / T_{v_{0}} V_{E, r e g} \simeq H^{0}\left(\mathcal{E} x t_{\mathcal{O}_{Y}}^{1}(E, E)\right) \simeq H^{0}\left(L^{*}\right)
$$


For question (ii), let $Z$ be an algebraic variety and $\mathcal{F}$ a coherent sheaf on $Z \times Y$, flat on $Z$. Let $z_{0} \in Z$ be such that $\mathcal{F}_{z_{0}} \simeq E$. So we get a morphism

$$
\phi:\left(Z, z_{0}\right) \longrightarrow\left(V_{E}, v_{0}\right)
$$

(where $\left(Z, z_{0}\right)$ is the germ defined by $Z$ and $z_{0}$ ). We say that $\mathcal{E}$ is a good family if $\mathcal{F}_{\mid Z \times C}$ is flat on $Z$ (or equivalently locally free). We show in 3.2 that in this case $\phi$ is a morphism to $V_{E \text {,reg }}$.

1.1.3. The case of sheaves on ribbons - Deformations of ribbons - We first give a general idea of the way question (iii') can be treated: we consider a deformation of $Y$, i.e. a flat morphism $\pi: \mathrm{e} \rightarrow S$, and $P \in S$, such that

$-S$ is a smooth curve.

$-\mathcal{C}_{P} \simeq Y$.

- for every $s \in S, \mathcal{C}_{s}$ is a projective curve.

We suppose that $E$ is a stable quasi locally free sheaf of rigid type. Let $\tau: \mathbb{M} \rightarrow S$ be the relative moduli space of stable sheaves with Hilbert polynomial $P_{E}$ (such that for every $s \in S$, $\mathbb{M}_{s}$ is the moduli space of stable sheaves on $\mathcal{C}_{s}$ with Hilbert polynomial $P_{E}$, cf. [15], [16]). We find that a suitable neighbourhood $U$ of $E$ in $\mathbb{M}_{P}$ is deformed in two non intersecting open subsets in two components of the fibres $\mathbb{M}_{s}, s \neq P$. The non reduced structure of $U$ comes from the fact that it is the "limit" of varieties with two connected components.

We have something similar for question (iii). More precisely suppose that $\operatorname{deg}(L)<0$. We will use for $\pi$ a maximal reducible deformation of $Y$, i.e.

- $\mathrm{C}$ is a reduced algebraic variety with two irreducible components $\mathrm{C}_{1}, \mathrm{C}_{2}$.

- For $i=1,2$, let $\pi_{i}: \mathcal{C}_{i} \rightarrow S$ be the restriction of $\pi$. Then $\pi_{i}^{-1}(P)=C$ and $\pi_{i}$ is a flat family of smooth irreducible projective curves.

- For every $s \in S \backslash\{P\}$, the components $\mathrm{C}_{1, s}, \mathrm{C}_{2, s}$ of $\mathcal{C}_{s}$ meet transversally.

For every $s \in S \backslash\{P\}, \mathcal{C}_{1, s}$ and $\mathcal{C}_{2, s}$ meet in exactly - $\operatorname{deg}(L)$ points. It is proved in [11] that such a deformation exists if $L$ can be written as $L=\mathcal{O}_{C}\left(-P_{1}-\cdots-P_{k}\right)$, for distinct points $P_{1}, \ldots, P_{k}$ of $C$. In this case we can construct $\mathcal{C}$ such that if $z$ is the closure of the set of intersections points of the components $\mathrm{C}_{1, s}, \mathrm{C}_{2, s}$ of $\mathrm{C}_{s}, s \neq P$, we have

$$
z \cap C=\left\{P_{1}, \ldots, P_{k}\right\} \text {. }
$$

In this way we define a one dimensional subspace $\Delta \subset H^{0}\left(L^{*}\right)$.

Let $E$ be a quasi locally free sheaf of rigid type on $Y$. Let $\mathcal{E}$ be a coherent sheaf on $\mathcal{C}$, flat on $S$ and such that $\mathcal{E}_{\mid Y}=E$. Suppose that $E$ is locally isomorphic to $r \mathcal{O}_{Y} \oplus \mathcal{O}_{C}$ with $r>0$. Then we from [1] there are two possibilities: for every $s \in S \backslash\{P\}$ in a neighbourhood of $P$

1) $\mathcal{E}_{s \mid \mathcal{e}_{1, s} \backslash z}$ is locally free of rank $r$ and $\mathcal{E}_{s \mid \mathcal{C}_{2, s} \backslash z}$ is locally free of rank $r+1$,

2) $\mathcal{E}_{s \mid \mathcal{E}_{1, s} \backslash z}$ is locally free of rank $r+1$ and $\mathcal{E}_{s \mid \mathcal{E}_{2, s} \backslash z}$ is locally free of rank $r$,

(that is: $\mathcal{E}$ is of rank $r$ on one of the components of $\mathcal{C}$ and of rank $r+1$ on the other). So we see that $E$ can be deformed in two distinct ways to sheaves on the reduced curves with two components.

Remarks: 1 - It is proved in [11] that given a quasi locally free sheaf $E$ on $Y$ (i.e. a sheaf locally isomorphic to a direct sum $\left.a \mathcal{O}_{Y} \oplus b \mathcal{O}_{C}\right)$, there exists a smooth curve $S^{\prime}, P^{\prime} \in S^{\prime}$, a 
morphism $f: S^{\prime} \rightarrow S$ such that $f\left(P^{\prime}\right)=P$, with non zero tangent map at $P^{\prime}$, and a coherent sheaf $\mathcal{E}^{\prime}$ on $f^{*} \mathcal{C}$ flat on $S^{\prime}$ and such that $\mathcal{E}_{P^{\prime}}^{\prime}=E$, i.e. $E$ can be deformed to sheaves on the reduced curves with two components.

2- In [6] many examples of non-empty moduli spaces of stable sheaves containing quasi locally free sheaves of rigid type are given.

1.1.4. Kodä̈ra-Spencer elements - Let $\mathcal{E}_{1}, \mathcal{E}_{2}$ be coherent sheaves on $\mathcal{C}$, flat on $S$, and such that $\mathcal{E}_{1, P}=\mathcal{E}_{2, P}=E$. Then we define

$$
\omega_{\mathcal{E}_{1}, \mathcal{E}_{2}} \in \operatorname{Ext}_{\mathcal{O}_{Y}}^{1}(E, E)
$$

(cf. 2.3): on the second neighbourhood $Y_{2}$ of $Y$ in $\mathcal{C}$ we have exact sequences

$$
0 \longrightarrow E \longrightarrow \mathcal{E}_{i \mid Y_{2}} \longrightarrow E \longrightarrow 0
$$

for $i=1,2$, and associated elements $\sigma_{i} \in \operatorname{Ext}_{\mathcal{O}_{Y_{2}}}^{1}(E, E)$. The difference $\omega_{\mathcal{E}_{1}, \mathcal{E}_{2}}=\sigma_{1}-\sigma_{2}$ lies in $\operatorname{Ext}_{\mathcal{O}_{Y}}^{1}(E, E)$ (this construction generalises that of the Kodaïra-Spencer morphism for sheaves on products $X \times Y)$.

We then consider two cases

\section{Case A}

$-\mathcal{E}_{s}^{[1]}$ is of rank $r$ on $\mathcal{C}_{2, s} \backslash \mathcal{Z}$ and of rank $r+1$ on $\mathcal{C}_{1, s} \backslash Z$.

$-\mathcal{E}_{s}^{[2]}$ is of rank $r+1$ on $\mathcal{C}_{2, s} \backslash \mathcal{Z}$ and of rank $r$ on $\mathcal{C}_{1, s} \backslash \mathcal{Z}$.

\section{Case B}

$-\mathcal{E}_{s}^{[1]}$ and $\mathcal{E}_{s}^{[2]}$ are of rank $r$ on $\mathcal{C}_{2, s} \backslash \mathcal{Z}$ and of rank $r+1$ on $\mathcal{C}_{1, s} \backslash \mathcal{Z}$.

Let

$$
\phi: \operatorname{Ext}_{\mathcal{O}_{Y}}^{1}(E, E) \longrightarrow H^{0}\left(\mathcal{E} x t_{\mathcal{O}_{Y}}^{1}(E, E)\right) \simeq H^{0}\left(L^{*}\right)
$$

be the canonical morphism. Recall that its kernel $H^{1}(\mathcal{E} n d(E))$ corresponds to good deformations of $E$, or deformations parametrised by a reduced variety (cf. 1.1.2). Then we have (theorem 5.2.1)

Theorem : $\mathbf{1}-$ In case $A, \phi\left(\omega_{\mathcal{E}^{[1]}, \mathcal{E}^{[2]}}\right)$ generates $\Delta$.

$\mathbf{2}-$ in case $B$, we have $\phi\left(\omega_{\mathcal{E}^{[1]}, \mathcal{E}^{[2]}}\right)=0$.

This means that the "non-reduced part" of the deformation of $E$, in $T_{v_{0}} V_{E} / T_{v_{0}} V_{E, r e g}$, corresponds to some parameter of the deformation of $Y$ to reduced curves with two components.

1.1.5. The case of moduli spaces of stable sheaves - Let $E$ be a stable quasi locally free sheaf of rigid type on $Y$. Let $\tau: \mathbb{M} \rightarrow S$ be the relative moduli space of stable sheaves with Hilbert polynomial $P_{E}$ (such that for every $s \in S, \mathbb{M}_{s}$ is the moduli space of stable sheaves on $\mathcal{C}_{s}$ with Hilbert polynomial $P_{E}$ ). For $s \in S \backslash\{P\}$, let $U_{1, s}$ (resp. $U_{2, s}$ ) be the open subset of $\mathbb{M}_{s}$ corresponding to linked sheaves (cf. 4.2) of rank $r$ on $\mathcal{C}_{1, s}$ and of rank $r+1$ on $\mathcal{C}_{2, s}$ (resp. of rank $r+1$ on $\mathcal{C}_{1, s}$ and of rank $r$ on $\left.\mathcal{C}_{2, s}\right)$. 
For $i=1,2$, let $\mathbf{U}_{i}=\bigcup_{s \in S \backslash\{P\}} U_{i, s}$. It is a smooth open subset of $\mathbb{M}$. Let $\mathbb{U}_{i} \subset \mathbb{M}$ be the closure of $\mathbf{U}_{i}$ and $\tau_{i}: \mathbb{U}_{i} \rightarrow S$ the restriction of $\tau$. Let $\mathbb{U}=\mathbb{U}_{1} \cup \mathbb{U}_{2}$, and $\bar{\tau}: \mathbb{U} \rightarrow S$ the restriction of $\tau$. Then from [11], $\bar{\tau}^{-1}(P) \subset \mathbb{M}_{P}$ contains the points corresponding to stable quasi locally free sheaves of rigid type.

The preceding theorem suggests the following natural

Conjecture: $1-\mathbb{U}_{i}$ is smooth and $\tau_{i}^{-1}(P)$ is smooth at $E$, i.e. around $E$, we have $\tau_{i}^{-1}(P)=\left(\mathbb{M}_{P}\right)_{\text {reg }}$.

$2-\mathbb{U}_{1}, \mathbb{U}_{2}$ intersect transversally, and the image of the composition

$$
T_{E}\left(\tau_{i}^{-1}(P)\right) \longrightarrow T_{E} \mathbb{M}_{P}=\operatorname{Ext}_{\mathcal{O}_{Y}}^{1}(E, E) \longrightarrow H^{0}\left(\mathcal{E} x t_{\mathcal{O}_{Y}}^{1}(E, E)\right)=H^{0}\left(L^{*}\right)
$$

is $\Delta$.

\subsection{Unstable RANK- 2 Vector BUNDLES ON $\mathbb{P}_{2}$}

The following results are proved in [18]:

Let $E$ be a rank-2 vector bundle on $\mathbb{P}_{2}$ such that $c_{1}(E)=0$. Let $d(E)$ be the largest integer such that $h^{0}(E(-d(E)))>0(E$ is stable if and only $d(E)<0)$.

Let $d, c_{2}$ be integers, such that $d \geq 3$ and $d^{2}+c_{2}>0$. Let $B(d)$ be the set of isomorphism classes of rank-2 vector bundles $E$ such that $c_{2}(E)=c_{2}$ and $d(E)=d$.

There is a natural structure of smooth irreducible algebraic variety on $B(d)$, and

$\operatorname{dim}(B(d))=3\left(d^{2}+c_{2}\right)-1$. Moreover, the subset $U$ of $B(d)$ consisting of bundles $E$ such that the deformations of $E$ are rank-2 bundles $F$ such that $d(F)=d$ is non-empty and open.

We have for $E \in B(d)$

$$
\operatorname{dim}\left(\operatorname{Ext}_{\mathcal{O}_{\mathbb{P}_{2}}}^{1}(E, E)\right)=4\left(d^{2}+c_{2}\right)-1>\operatorname{dim}(B(d)) .
$$

If $E \in U, V_{E, \text { reg }}$ is smooth of dimension $\operatorname{dim}(B(d))$, and $V_{E}$ is not reduced.

Let $Z$ be an algebraic variety, $z_{0} \in Z$, and $\mathcal{F}$ a coherent sheaf on $Z \times \mathbb{P}_{2}$, flat on $Z$ and such that $\mathcal{F}_{z} \in U$ for every closed point $z \in Z$. Let $E=\mathcal{F}_{z_{0}}$. Then we have a morphism

$$
\phi:\left(Z, z_{0}\right) \rightarrow\left(V_{E}, v_{0}\right)
$$

such that $\left(\phi \times I_{\mathbb{P}_{2}}\right)^{*}\left(\mathcal{E}_{E}\right) \simeq \mathcal{F}$.

Let $p_{Z}: Z \times \mathbb{P}_{2} \rightarrow Z, p_{2}: Z \times \mathbb{P}_{2} \rightarrow \mathbb{P}_{2}$ be the projections, and

$$
\mathcal{L}=p_{Z *}\left(\mathcal{F} \otimes p_{2}^{*}\left(\mathcal{O}_{\mathbb{P}_{2}}(-d)\right)\right)
$$

For every closed point $z \in Z, \mathcal{L}_{z} \otimes_{\mathcal{O}_{Z, z}} \mathbb{C}(z)$ has dimension one. We say that $\mathcal{F}$ has pure type $d$ if $\mathcal{L}$ is locally free (or equivalently if $\mathcal{L}$ is flat on $Z$ ). If $\mathcal{L}$ is pure of type $d$ then $\phi$ is a morphism to $V_{E, r e g}$, i.e. we have a result which is similar to that of 1.1.2. 


\subsection{OUtLine OF THE PAPER}

Section 2 contains definitions and properties of primitive multiple curves of any multiplicity, with some particular results in multiplicity 2 (primitive multiple curves of multiplicity 2 are also called double curves or ribbons). This section contains also a description of the generalisation of the Kodaïra-Spencer morphism that is used here.

Section 3 is devoted to the study of quasi locally free sheaves of rigid type on a primitive multiple curve, and to their deformations. In particular we give an answer to question (ii) of 1.1.1. Some results are valid in any multiplicity.

In section 4 we recall some definitions concerning maximal reducible deformations of ribbons, i.e. deformations to reduced curves with two components intersecting transversally. We recall also some results about deformations of quasi locally free sheaves of rigid type on ribbons to sheaves on the reduced curves with two components.

In section 5 we prove the main result of this paper, i.e. the theorem in 1.1.4.

\section{PRELiminARIES}

\subsection{Primitive Multiple CURVES AND QUASi LOCAlly FreE SHEAVES}

(cf. [1], [2], 4], 5], 6], [7], 8], [12]).

2.1.1. Definitions - Let $C$ be a smooth connected projective curve. A multiple curve with support $C$ is a Cohen-Macaulay scheme $Y$ such that $Y_{\text {red }}=C$.

Let $n$ be the smallest integer such that $Y=C^{(n-1)}, C^{(k-1)}$ being the $k$-th infinitesimal neighbourhood of $C$, i.e. $\mathcal{J}_{C^{(k-1)}}=\mathcal{J}_{C}^{k}$. We have a filtration $C=C_{1} \subset C_{2} \subset \cdots \subset C_{n}=Y$ where $C_{i}$ is the biggest Cohen-Macaulay subscheme contained in $Y \cap C^{(i-1)}$. We call $n$ the multiplicity of $Y$.

We say that $Y$ is primitive if, for every closed point $x$ of $C$, there exists a smooth surface $S$, containing a neighbourhood of $x$ in $Y$ as a locally closed subvariety. In this case, $L=\mathcal{J}_{C} / \mathcal{J}_{C_{2}}$ is a line bundle on $C$ and we have $\mathcal{J}_{C_{j}}=\mathcal{J}_{C}^{j}, \mathcal{J}_{C_{j}} / \mathcal{J}_{C_{j+1}}=L^{j}$ for $1 \leq j<n$. We call $L$ the line bundle on $C$ associated to $Y$. Let $P \in C$. Then there exist elements $y, t$ of $m_{S, P}$ (the maximal ideal of $\left.\mathcal{O}_{S, P}\right)$ whose images in $m_{S, P} / m_{S, P}^{2}$ form a basis, and such that for $1 \leq i<n$ we have $\mathcal{J}_{C_{i}, P}=\left(y^{i}\right)$.

The simplest case is when $Y$ is contained in a smooth surface $S$. Suppose that $Y$ has multiplicity $n$. Let $P \in C$ and $f \in \mathcal{O}_{S, P}$ a local equation of $C$. Then we have $\mathcal{J}_{C_{i}, P}=\left(f^{i}\right)$ for $1<j \leq n$, in particular $I_{Y, P}=\left(f^{n}\right)$, and $L=\mathcal{O}_{C}(-C)$.

For any $L \in \operatorname{Pic}(C)$, the trivial primitive curve of multiplicity $n$, with induced smooth curve $C$ and associated line bundle $L$ on $C$ is the $n$-th infinitesimal neighbourhood of $C$, embedded by the zero section in the dual bundle $L^{*}$, seen as a surface.

We will write $\mathcal{O}_{n}=\mathcal{O}_{C_{n}}$ and we will see $\mathcal{O}_{i}$ as a coherent sheaf on $C_{n}$ with schematic support $C_{i}$ if $1 \leq i<n$. 
2.1.2. Invariants of sheaves and canonical filtrations - If $\mathcal{E}$ is a coherent sheaf on $Y$ one defines its generalised rank $R(\mathcal{E})$ and generalised degree $\operatorname{Deg}(\mathcal{E})$ (cf. [6], 3-): take any filtration of $\mathcal{E}$

$$
0=\mathcal{F}_{n} \subset \mathcal{F}_{n-1} \subset \cdots \subset \mathcal{F}_{0}=\mathcal{E}
$$

by subsheaves such that $\mathcal{F}_{i} / \mathcal{F}_{i+1}$ is concentrated on $C$ for $0 \leq i<n$, then

$$
R(\mathcal{E})=\sum_{i=0}^{n-1} \operatorname{rk}\left(\mathcal{F}_{i} / \mathcal{F}_{i+1}\right) \quad \text { and } \quad \operatorname{Deg}(\mathcal{E})=\sum_{i=0}^{n-1} \operatorname{deg}\left(\mathcal{F}_{i} / \mathcal{F}_{i+1}\right) .
$$

Let $\mathcal{O}_{Y}(1)$ be a very ample line bundle on $Y$. Then the Hilbert polynomial of $\mathcal{E}$ is

$$
P_{\mathcal{E}}(m)=R(\mathcal{E}) \operatorname{deg}\left(\mathcal{O}_{C}(1)\right) m+\operatorname{Deg}(\mathcal{E})+R(\mathcal{E})(1-g)
$$

(where $g$ is the genus of $C$ ).

The first canonical filtration of $\mathcal{E}$

$$
0=\mathcal{E}_{n} \subset \mathcal{E}_{n-1} \subset \cdots \subset \mathcal{E}_{0}=\mathcal{E}
$$

is defined as follows: for $0 \leq i \leq n$, we have $\mathcal{E}_{i}=J_{C}^{i} \mathcal{E}$. For $0 \leq i<n$, the sheaf

$G_{i}(\mathcal{E})=\mathcal{E}_{i} / \mathcal{E}_{i+1}$ is concentrated on $C$. The same definition applies if $\mathcal{E}$ is a coherent sheaf on a non-empty open subset of $Y$. The pair

$$
\sigma(\mathcal{E})=\left(\left(\operatorname{rg}\left(G_{0}(\mathcal{E})\right), \ldots, \operatorname{rg}\left(G_{n-1}(\mathcal{E})\right),\left(\operatorname{deg}\left(G_{0}(\mathcal{E})\right), \ldots, \operatorname{deg}\left(G_{n-1}(\mathcal{E})\right)\right)\right.\right.
$$

is called the complete type of $\mathcal{E}$.

Let $P \in C$ and $M$ a $\mathcal{O}_{Y, P}$-module of finite type. We also define the first canonical filtration of $M$

$$
0=M_{n} \subset M_{n-1} \subset \cdots \subset M_{0}=M
$$

as: $M_{i}=\mathcal{J}_{C, P}^{i} M$. The quotients $G_{i}(M)=M_{i} / M_{i+1}$ are $\mathcal{O}_{C, P}$-modules. The generalised rank of $M$ is $R(M)=\sum_{i=0}^{n-1} \operatorname{rk}\left(G_{i}(M)\right)$.

Let $X$ be an algebraic variety and $\mathbb{E}$ a coherent sheaf on $X \times Y$, flat on $X$. We can also define the first canonical filtration of $\mathbb{E}$

$$
0=\mathbb{E}_{n} \subset \mathbb{E}_{n-1} \subset \cdots \subset \mathbb{E}_{0}=\mathbb{E}
$$

by $\mathbb{E}_{i}=p_{Y}^{*}\left(\mathcal{J}_{C}^{i}\right) \mathbb{E}$.

The second canonical filtration of $\mathcal{E}$

$$
0=\mathcal{E}^{(0)} \subset \mathcal{E}^{(1)} \subset \cdots \subset \mathcal{E}^{(n)}=\mathcal{E}
$$

is defined as follows: for $0 \leq i \leq n$ and $P \in C, \mathcal{E}_{P}^{(i)}$ is the set of $u \in \mathcal{E}_{P}$ such that $\mathcal{J}_{C, P}^{i} u=0$. For $1 \leq i \leq n$, the sheaf $G^{(i)}(\mathcal{E})=\mathcal{E}^{(i)} / \mathcal{E}^{(i-1)}$ is concentrated on $C$.

Let $P \in C$ and $M$ a $\mathcal{O}_{Y, P}$-module of finite type. We also define the second canonical filtration of $M$

in the obvious way.

$$
0=M^{(0)} \subset M^{(1)} \subset \cdots \subset M^{(n)}=M
$$


2.1.3. The case of double curves - If $n=2$, let $\mathcal{E}$ be a coherent sheaf on $Y=C_{2}$. Then we have canonical exact sequences

$$
0 \longrightarrow \mathcal{E}_{1} \longrightarrow \mathcal{E} \longrightarrow \mathcal{E}_{\mid C} \longrightarrow 0, \quad 0 \longrightarrow \mathcal{E}^{(1)} \longrightarrow \mathcal{E} \longrightarrow \mathcal{E}^{(2)} \simeq \mathcal{E}_{1} \otimes L^{*} \longrightarrow 0 .
$$

2.1.4. Quasi locally free sheaves - Let $P \in C$ and $M$ a $\mathcal{O}_{Y, P}$-module of finite type. We say that $M$ is quasi free if there exist integers $m_{i} \geq 0,1 \leq i \leq n$, such that $M \simeq \bigoplus_{i=1}^{n} m_{i} \mathcal{O}_{i, P}$. These integers are uniquely determined. In this case we say that $M$ is of type $\left(m_{1}, \ldots, m_{n}\right)$. We have $R(M)=\sum_{i=1}^{n} i . m_{i}$.

Let $\mathcal{E}$ be a coherent sheaf on a non-empty open subset $V \subset Y$. We say that $\mathcal{E}$ est quasi locally free at a point $P$ of $V$ if there exists a neighbourhood $U \subset V$ of $P$ and integers $m_{i} \geq 0,1 \leq i \leq n$, such that for every $Q \in U, \mathcal{E}_{Q}$ is quasi free of type $m_{1}, \ldots, m_{n}$. The integers $m_{1}, \ldots, m_{n}$ are uniquely determined and depend only of $\mathcal{E}$, and $\left(m_{1}, \ldots, m_{n}\right)$ is called the type of $\mathcal{E}$.

We say that $\mathcal{E}$ est quasi locally free if it is quasi locally free at every point of $V$.

The following conditions are equivalent:

(i) $\mathcal{E}_{P}$ is quasi locally free at $P$.

(ii) The $\mathcal{O}_{C, P}$-modules $G_{i}\left(\mathcal{E}_{P}\right)$ are free.

The following conditions are equivalent:

(i) $\mathcal{E}$ is quasi locally free.

(ii) the sheaves $G_{i}(\mathcal{E})$ are locally free on $C$.

\subsection{InFINITESIMAL DEFORMATIONS OF COHERENT SHEAVES}

2.2.1. Deformations of sheaves - Let $X$ be a projective algebraic variety and $E$ a coherent sheaf on $X$. A deformation of $E$ is a quadruplet $\mathcal{D}=\left(S, s_{0}, \mathcal{E}, \alpha\right)$, where $\left(S, s_{0}\right)$ is the germ of an analytic variety, $\mathcal{E}$ is a coherent sheaf on $S \times X$, flat on $S$, and $\alpha$ an isomorphism $\mathcal{E}_{s_{0}} \simeq E$. If there is no risk of confusion, we also say that $\mathcal{E}$ is an infinitesimal deformation of $E$. Let $\left.Z_{2}=\operatorname{spec}(\mathbb{C} \mid t] /\left(t^{2}\right)\right)$. When $S=Z_{2}$ and $s_{0}$ is the closed point $*$ of $Z_{2}$, we say that $\mathcal{D}$ is an infinitesimal deformation of $E$. Isomorphisms of deformations of $E$ are defined in an obvious way. If $f:\left(S^{\prime}, s_{0}^{\prime}\right) \rightarrow\left(S, s_{0}\right)$ is a morphism of germs, the deformation $f^{\#}(\mathcal{D})$ is defined as well. A deformation $\mathcal{D}=\left(S, s_{0}, \mathcal{E}, \alpha\right)$ is called semi-universal if for every deformation $\mathcal{D}^{\prime}=\left(S^{\prime}, s_{0}^{\prime}, \mathcal{E}^{\prime}, \alpha^{\prime}\right)$ of $E$, there exists a morphism $f:\left(S^{\prime}, s_{0}^{\prime}\right) \rightarrow\left(S, s_{0}\right)$ such that $f^{\#}(\mathcal{D}) \simeq \mathcal{D}^{\prime}$, and if the tangent map $T_{s_{0}^{\prime}} S^{\prime} \rightarrow T_{s_{0}} S$ is uniquely determined. There always exists a semiuniversal deformation of $E$ (cf. [17], theorem I).

Let $\mathcal{E}$ be an infinitesimal deformation of $E$. Let $p_{X}$ denote the projection $Z_{2} \times X \rightarrow X$. Then there is a canonical exact sequence

$$
0 \longrightarrow E \longrightarrow p_{X *}(\mathcal{E}) \longrightarrow E \longrightarrow 0
$$

i.e. an extension of $E$ by itself. In fact, by associating this extension to $\mathcal{E}$ one defines a bijection between the set of isomorphism classes of infinitesimal deformations of $E$ and the set of isomorphism classes of extensions of $E$ by itself, i.e. $\operatorname{Ext}_{\mathcal{O}_{X}}^{1}(E, E)$. 
2.2.2. Kodaïra-Spencer morphism - Let $\mathcal{D}=\left(S, s_{0}, \mathcal{E}, \alpha\right)$ be a deformation of $E$, and $X_{s_{0}}^{(2)}$ the infinitesimal neighbourhood of order 2 of $X_{s_{0}}=\left\{s_{0}\right\} \times X$ in $S \times X$. Then we have an exact sequence on $X_{s_{0}}^{(2)}$

$$
0 \longrightarrow T_{s_{0}} S \otimes E \longrightarrow \mathcal{E} / m_{s}^{2} \mathcal{E}=\mathcal{E}_{\mid X_{s_{0}}^{(2)}} \longrightarrow E \longrightarrow 0
$$

By taking the direct image by $p_{X}$ we obtain the exact sequence on $X$

$$
0 \longrightarrow T_{s_{0}} S \otimes E \longrightarrow F \longrightarrow E \longrightarrow 0
$$

hence a linear map

$$
\omega_{s_{0}}: T_{s_{0}} S \longrightarrow \operatorname{Ext}_{\mathcal{O}_{X}}^{1}(E, E)
$$

which is called the Kodä̈ra-Spencer morphism of $\mathcal{E}$ at $s_{0}$.

We say that $\mathcal{E}$ is a complete deformation if $\omega_{s_{0}}$ is surjective. If $\mathcal{D}$ is a semi-universal deformation, $\omega_{s_{0}}$ is an isomorphism.

\subsection{Generalisation of the KodaÏra-Spencer morphism}

Let $S$ be a smooth curve and $s_{0} \in S$ a closed point. Let $\rho: \mathcal{X} \rightarrow S$ be a flat projective morphism of algebraic varieties. Let $Y=\rho^{-1}\left(s_{0}\right)$. It is a projective variety. Let $\mathcal{E}$, $\mathcal{E}^{\prime}$ be coherent sheaves on $\mathcal{X}$, flat on $S$, such that there exists an isomorphism $\mathcal{E}_{\mid Y} \simeq \mathcal{E}_{\mid Y}^{\prime}$. Let $E=\mathcal{E}_{\mid Y}$. Let $Y_{2}$ be the second infinitesimal neighbourhood of $Y$ in $X$. If $s_{0}^{(2)}$ is the second infinitesimal neighbourhood of $s_{0}$ in $S$, we have $Y_{2}=\rho^{-1}\left(s_{0}^{(2)}\right)$. The ideal sheaf $\mathcal{J}_{Y}$ of $Y$ in $Y_{2}$ is isomorphic to $\mathcal{O}_{Y}$. We have canonical exact sequences

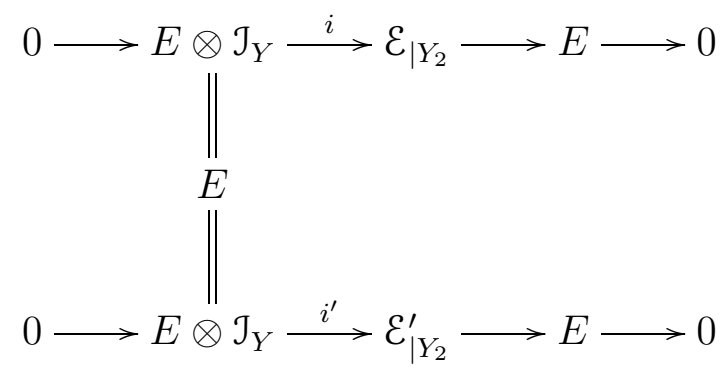

(the injectivity of $i$ and $i^{\prime}$ follows easily from the flatness of $\mathcal{E}, \mathcal{E}^{\prime}$ over $S$ ). Let $\sigma, \sigma^{\prime} \in \operatorname{Ext}_{\mathcal{O}_{Y_{2}}}^{1}(E, E)$ correspond to these extensions.

Let $0 \rightarrow E \rightarrow \mathcal{F} \rightarrow E \rightarrow 0$ be an exact sequence of coherent sheaves on $Y_{2}$. The canonical morphism $\mathcal{F} \otimes \mathcal{J}_{Y} \rightarrow \mathcal{F}$ induces a morphism $E \otimes \mathcal{J}_{Y} \rightarrow E$, which vanishes if and only if $\mathcal{F}$ is concentrated on $Y$. In this way we get an exact sequence

$$
0 \longrightarrow \operatorname{Ext}_{\mathcal{O}_{Y}}^{1}(E, E) \longrightarrow \operatorname{Ext}_{\mathcal{O}_{Y_{2}}}^{1}(E, E) \stackrel{p}{\longrightarrow} \operatorname{End}(E)
$$

We have $p(\sigma)=p\left(\sigma^{\prime}\right)=I_{E}$. So we have

$$
\omega_{\mathcal{E}, \mathcal{E}^{\prime}}=\sigma-\sigma^{\prime} \in \operatorname{Ext}_{\mathcal{O}_{Y}}^{1}(E, E) .
$$

Connections with the Kodaïra-Spencer morphism - Suppose the $\mathcal{X}$ is the trivial family: $X=Y \times S$. Let

$$
\omega_{s_{0}}: T_{s_{0}} S \longrightarrow \operatorname{Ext}_{\mathcal{O}_{Y}}^{1}(E, E)
$$


be the Kodaïra-Spencer morphism of $\mathcal{E}$. Suppose that $\mathcal{E}^{\prime}$ is the trivial family: $\mathcal{E}^{\prime}=p_{Y}^{*}(E)$ (where $p_{Y}$ is the projection $Y \times S \rightarrow S$ ). The isomorphism $\mathcal{J}_{Y} \simeq \mathcal{O}_{Y}$ is defined by the choice of a generator $t$ of the maximal ideal of $s_{0}$ in $S$. Let $u$ the associated element of $T_{s_{0}} S$. Then we have $\rho\left(\sigma-\sigma^{\prime}\right)=\omega_{s_{0}}(u)$.

\section{QUASI LOCALLY FREE SHEAVES OF RIGID TYPE}

We keep the notations of 2.1 .

\subsection{Definitions And BASiC PROperties}

A quasi locally free sheaf $\mathcal{E}$ on $Y$ is called of rigid type if it is locally free or locally isomorphic to $a \mathcal{O}_{n} \oplus \mathcal{O}_{k}$ for some integers $a \geq 0,1 \leq k<n$. The set of isomorphism classes of quasi locally free sheaves of rigid type of fixed complete type (cf. [2.1.2) is an open family (cf. [6], 6-): let $X$ be an algebraic variety, $\mathbb{E}$ a coherent sheaf on $X \times Y$, flat on $X$, and $x \in X$ a closed point. Suppose that $\mathbb{E}_{x}$ is quasi locally free of rigid type. Then there exists an open subset $U$ of $X$ containing $x$ such that, for every $x^{\prime} \in U, \mathbb{E}_{x^{\prime}}$ is quasi locally free and $\sigma\left(\mathbb{E}_{x^{\prime}}\right)=\sigma\left(\mathbb{E}_{x}\right)$.

More generally, let $\mathcal{E}$ be a quasi locally free sheaf on $Y$, locally isomorphic to $a \mathcal{O}_{n} \oplus b \mathcal{O}_{k}$, with $a, b>0,1 \leq k<n$. By [6], prop. 5.1, there exists a vector bundle $\mathbb{E}$ on $Y$ and a surjective morphism

$$
\phi: \mathbb{E} \longrightarrow \mathcal{E}
$$

inducing an isomorphism $\mathbb{E}_{\mid C} \simeq \mathcal{E}_{\mid C}$. Let $\mathcal{L}=\operatorname{ker}(\phi)$. By [6], lemme $5.2, \mathcal{L}$ is a vector bundle of rank $b$ on $C_{n-k}$. Let $P \in C$, and $z \in \mathcal{O}_{Y, P}$ an equation of $C$. At $P$ the exact sequence $0 \rightarrow \mathcal{L} \rightarrow \mathbb{E} \rightarrow \mathcal{E} \rightarrow 0$ is isomorphic to the trivial one

$$
0 \longrightarrow\left(z^{k}\right) \otimes \mathbb{C}^{b} \simeq \mathcal{O}_{n-k, P} \otimes \mathbb{C}^{b} \longrightarrow \mathcal{O}_{n, P} \otimes\left(\mathbb{C}^{a} \oplus \mathbb{C}^{b}\right) \longrightarrow\left(\mathcal{O}_{n, P} \otimes \mathbb{C}^{a}\right) \oplus\left(\mathcal{O}_{k, P} \otimes \mathbb{C}^{b}\right) \longrightarrow 0 .
$$

\subsubsection{Lemma: There is a canonical isomorphism}

$$
\mathcal{L}_{\mid C_{k}} \simeq\left(\mathcal{E}^{(k)} / \mathcal{E}_{n-k}\right) \otimes \mathcal{J}_{C}^{k}
$$

Proof. Let $P \in C, z \in \mathcal{O}_{Y, P}$ an equation of $C$ and $u \in \mathcal{E}_{P}^{(k)}$. Let $v \in \mathbb{E}_{P}$ be such that $\phi_{P}(v)=u$. Then we have

$$
\phi_{P}\left(z^{k} v\right)=z^{k} u=0,
$$

hence $z^{k} v \in \mathcal{L}_{P}$. If $v^{\prime} \in \mathbb{E}_{P}$ is such that $\phi_{P}\left(v^{\prime}\right)=u$, we have $w=v^{\prime}-v \in \mathcal{L}_{P}$, hence the image of $z^{k} v^{\prime}=z^{k} v+z^{k} w$ in $\mathcal{L}_{P} / z^{k} \mathcal{L}_{P}$ is the same as that of $z^{k} v$. By associating $z^{k} v$ to $u \otimes z^{k}$ we define a morphism $\bar{\theta}: \mathcal{E}^{(k)} \otimes \mathcal{J}_{C}^{k} \rightarrow \mathcal{L}_{\mid C_{k}}$. If $u \in \mathcal{E}_{n-k, P}$, let $u^{\prime} \in \mathcal{E}_{P}$ be such that $u=z^{n-k} u^{\prime}$. Let $v^{\prime} \in \mathbb{E}_{P}$ be such that $\phi_{P}\left(v^{\prime}\right)=u^{\prime}$. Then we can take $v=z^{n-k} v^{\prime}$, and then $z^{k} v=0$, hence $\bar{\theta}_{P}\left(u \otimes z^{k}\right)=0$. It follows that $\bar{\phi}$ induces a morphism

$$
\theta:\left(\mathcal{E}^{(k)} / \mathcal{E}_{n-k}\right) \otimes \mathcal{J}_{C}^{k} \longrightarrow \mathcal{L}_{\mid C_{k}}
$$

In the above description of the exact sequence $0 \rightarrow \mathcal{L} \rightarrow \mathbb{E} \rightarrow \mathcal{E} \rightarrow 0$, we have $\varepsilon_{P}^{(k)} / \mathcal{E}_{n-k, P}=\mathcal{O}_{k, P} \otimes \mathbb{C}^{b}$, and $\theta_{P}=\left(\mathcal{O}_{k, P} \otimes \mathbb{C}^{b}\right) \otimes\left(z^{k}\right) \rightarrow\left(z^{k}\right) \otimes \mathbb{C}^{b}$ is the identity morphism. Hence $\phi$ is an isomorphism. 
The sheaf $\mathcal{L}_{\mid C_{k}}$ is a vector bundle of rank $b$, on $C_{k}$ if $2 k \leq n$, and on $C_{n-k}$ if $2 k>n$.

3.1.2. Corollary: There is a canonical isomorphism

$$
\mathcal{E} x t_{\mathcal{O}_{Y}}^{1}(\mathcal{E}, \mathcal{E}) \simeq \mathcal{H} \operatorname{Com}\left(\left(\mathcal{E}^{(k)} / \mathcal{E}_{n-k}\right) \otimes \mathcal{J}_{C}^{k}, \mathcal{E}^{(k)} / \mathcal{E}_{n-k}\right)
$$

Proof. From the exact sequence $0 \rightarrow \mathcal{L} \rightarrow \mathbb{E} \rightarrow \mathcal{E} \rightarrow 0$, we deduce the exact sequence

$$
\mathcal{H} \operatorname{om}(\mathbb{E}, \mathcal{E}) \longrightarrow \mathcal{H} \operatorname{Com}(\mathcal{L}, \mathcal{E}) \longrightarrow \mathcal{E} x t_{\mathcal{O}_{Y}}^{1}(\mathcal{E}, \mathcal{E}) \longrightarrow 0
$$

and the result follows easily using local isomorphisms of $\mathcal{E}$ with $a \mathcal{O}_{n} \oplus b \mathcal{O}_{k}$ and lemma 3.1 .1 .

If $\mathcal{E}$ is of rigid type (i.e. if $b=1$ ), then $\mathcal{E}^{(k)} / \mathcal{E}_{n-k}$ is a line bundle on $C_{\inf (k, n-k)}$, and it follows that

$$
\mathcal{E} x t_{\mathcal{O}_{Y}}^{1}(\mathcal{E}, \mathcal{E}) \simeq \mathcal{H} \operatorname{Hom}\left(\mathcal{J}_{C \mid C_{\inf (k, n-k)}^{k}}, \mathcal{O}_{\inf (k, n-k)}\right)
$$

It follows that we have an exact sequence

$$
\begin{gathered}
0 \longrightarrow H^{1}(\mathcal{E} n d(\mathcal{E})) \longrightarrow \operatorname{Ext}_{\mathcal{O}_{Y}}^{1}(\mathcal{E}, \mathcal{E}) \longrightarrow \operatorname{Hom}\left(\mathcal{J}_{C \mid C_{\text {inf }(k, n-k)}^{k}}, \mathcal{O}_{\inf (k, n-k)}\right) \longrightarrow 0 \\
H^{0}\left(\mathcal{E} x t_{\mathcal{O}_{Y}}^{1}(\mathcal{E}, \mathcal{E})\right)
\end{gathered}
$$

3.1.3. The case of double curves - If $n=2$ we have $k=1$ and from corollary 3.1 .2

$$
\varepsilon x t_{\mathcal{O}_{Y}}^{1}(\varepsilon, \mathcal{E}) \simeq L^{*} \text {. }
$$

3.1.4. Remark: Let Let $X$ be an algebraic variety, $\mathbb{E}$ a coherent sheaf on $X \times Y$, flat on $X$, such that for every closed point $x \in X, \mathbb{E}_{x}$ is quasi locally free of rigid type. Let $x \in X$, and

$$
\omega_{x}(\mathbb{E}): T X_{x} \longrightarrow \operatorname{Ext}_{\mathcal{O}_{Y}}^{1}\left(\mathbb{E}_{x}, \mathbb{E}_{x}\right)
$$

the Kodaïra-Spencer morphism of $\mathbb{E}$. Let $X_{\text {red }}$ be the reduced subscheme associated to $X$. Then the image of Kodaïra-Spencer morphism of $\mathbb{E}_{X_{\text {red }} \times Y}$

$$
\omega_{x}\left(\mathbb{E}_{X_{\text {red }} \times Y}\right): T X_{\text {red }, x} \longrightarrow \operatorname{Ext}_{\mathcal{O}_{Y}}^{1}\left(\mathbb{E}_{x}, \mathbb{E}_{x}\right)
$$

is contained in $H^{1}\left(\varepsilon n d\left(\mathbb{E}_{x}\right)\right)$. Suppose that $\mathbb{E}$ is a complete deformation of $\mathbb{E}_{x}$ (i.e. $\omega_{x}(\mathbb{E})$ is surjective), and that $\mathbb{E}_{x}$ is simple. Then $\operatorname{im}\left(\omega_{x}\left(\mathbb{E}_{\mid X_{\text {red }} \times Y}\right)\right)=H^{1}\left(\mathcal{E} n d\left(\mathbb{E}_{x}\right)\right)$ (cf. [6], th. 6.10, cor. 6.11).

\subsection{FAMILIES OF QUASI LOCALly FREE SHEAVES OF RIGID TYPE}

Let $m_{1}, \ldots, m_{n}$ be non negative integers. Let $X$ be a connected algebraic variety, $U \subset X \times Y$ an open subset such that $p_{X}(U)=X$ (where $p_{X}$ is the projection $X \times Y \rightarrow X$ ) and $\mathbb{E}$ a coherent sheaf on $U$, flat on $X$, such that for every closed point $x \in X, \mathbb{E}_{x}$ is quasi locally free of type $\left(m_{1}, \ldots, m_{n}\right)$. We say that $\mathbb{E}$ is a good family if for $0 \leq i<n$ the sheaf $\mathbb{E}_{i} / \mathbb{E}_{i+1}$ on $(X \times C) \cap U$ is flat on $X$ (where $0=\mathbb{E}_{n} \subset \mathbb{E}_{n-1} \subset \cdots \subset \mathbb{E}_{0}=\mathbb{E}$ is the first canonical filtration of $\mathbb{E}$ ). It $\mathbb{E}$ is a good family then by [13], exp. IV, prop. 1.1 , for $0 \leq i<n, \mathbb{E}_{i}$ is a flat family of sheaves on $C_{n-i}$, and by [16], lemma $1.27, \mathbb{E}_{i} / \mathbb{E}_{i+1}$ is a vector bundle on $X \times C$. 
3.2.1. Theorem: 1 - The sheaf $\mathbb{E}$ is a good family if and only if it is locally isomorphic to $\bigoplus_{i=1}^{n} \mathcal{O}_{X \times C_{i}} \otimes \mathbb{C}^{m_{i}}$

If $\mathbb{E}$ is a good family on $X \times Y$, then for every $x \in X$ the image of Kodaïra-Spencer morphism of $\mathbb{E}$

$$
\omega_{x}(\mathbb{E}): T X_{x} \longrightarrow \operatorname{Ext}_{\mathcal{O}_{Y}}^{1}\left(\mathbb{E}_{x}, \mathbb{E}_{x}\right)
$$

is contained in $H^{1}\left(\mathcal{E} n d\left(\mathbb{E}_{x}\right)\right)$.

Proof. Suppose that $\mathbb{E}$ is locally isomorphic to $\bigoplus_{i=1}^{n} \mathcal{O}_{X \times C_{i}} \otimes \mathbb{C}^{m_{i}}$. Then it is obvious that the sheaves $\mathbb{E}_{i} / \mathbb{E}_{i+1}$ are vector bundles on $(X \times C) \cap U$, hence they are flat on $X$ and $\mathbb{E}$ is a good family. On the other hand, the local structure of $\mathbb{E}$ does not vary when $x$ varies, hence for every $P \in C$, the image of $\operatorname{im}\left(\omega_{x}(\mathbb{E})\right)$ in $\operatorname{Ext}_{\hat{O}_{Y, P}}^{1}\left(\mathbb{E}_{x, P}, \mathbb{E}_{x, P}\right)$ must be 0 , so $\operatorname{im}\left(\omega_{x}(\mathbb{E})\right) \subset H^{1}\left(\mathcal{E} n d\left(\mathbb{E}_{x}\right)\right)$.

Conversely, suppose that $\mathbb{E}$ is a good family. The proof that $\mathbb{E}$ is locally isomorphic to $\bigoplus_{i=1}^{n} \mathcal{O}_{X \times C_{i}} \otimes \mathbb{C}^{m_{i}}$ is similar to that of theor. 6.5 of [6]. We make an induction on $n$. The result for $n=1$ follows from [16], lemma 1.27. Suppose that it is true for $n-1 \geq 1$. We make an induction on $m_{n}$.

Suppose that $m_{n}=0$. Let $k$ be the smallest integer such that $m_{q}=0$ for $k+1 \leq q \leq n$. Then we have $k<n$, and for every $x \in X, \mathbb{E}_{x}$ is concentrated on $C_{k}$. Then $\mathbb{E}$ is concentrated on $\left(X \times C_{k}\right) \cap U$ : this follows easily by induction on $k$ from the exact sequence $0 \rightarrow \mathbb{E}_{1} \rightarrow \mathbb{E} \rightarrow \mathbb{E}_{(X \times C) \cap U} \rightarrow 0$, using the fact that $\mathbb{E}_{1}$ is flat on $X$. By the induction hypothesis (on $\left.\left(X \times C_{k}\right) \cap U\right), \mathbb{E}$ is locally isomorphic to $\bigoplus_{i=1}^{n} \mathcal{O}_{X \times C_{i}} \otimes \mathbb{C}^{m_{i}}$.

Suppose that the result is true for $m_{n}-1 \geq 0$. Let $P \in C, x \in X$ such that $Q=(x, P) \in U$. For every open subset $V \subset X \times Y$ and $x^{\prime} \in X$, let $V_{x^{\prime}}=V \cap(\{x\} \times Y)$. Let $Z \subset U$ be an open affine subset containing $Q$ such that there is an isomorphism

$$
\mathbb{E}_{x \mid Z_{x}} \simeq \bigoplus_{i=1}^{n} \mathcal{O}_{i \mid Z_{x}} \otimes \mathbb{C}^{m_{i}}
$$

and that $\Delta=p_{Y}^{*}\left(\mathcal{J}_{C}\right)$ (which is a line bundle on $\left.\left(X \times C_{n-1}\right) \cap U\right)$ is trivial on $\left(X \times C_{n-1}\right) \cap Z$. Let $\zeta \in H^{0}(\Delta)$ a section inducing an isomorphism $\Delta \simeq \mathcal{O}_{\left(X \times C_{n-1}\right) \cap Z}$. Let $\sigma \in H^{0}\left(\mathbb{E}_{x \mid Z_{x}}\right)$ defined by some non zero element of $\mathbb{C}^{m_{n}}$, and $\bar{\sigma} \in H^{0}\left(\mathbb{E}_{\mid Z}\right)$ extending $\sigma$. Then $\zeta^{n-1} \bar{\sigma} \in H^{0}\left(Z, \mathbb{E}_{n-1}\right)$, $\mathbb{E}_{n-1}$ is a vector bundle on $(X \times C) \cap Z$, and $s=\zeta^{n-1} \bar{\sigma}_{\mid Z_{x}}$ does not vanish on $Z_{x}$. Let $T \subset Z$ be the open subset where $s$ does not vanish. Let $x^{\prime} \in X$ such that $T_{x^{\prime}} \neq \emptyset$, and $W \subset T_{x^{\prime}}$ an open subset such that $\mathbb{E}_{\mid W} \simeq \bigoplus_{i=1}^{n} \mathcal{O}_{i \mid W} \times \mathbb{C}^{m_{i}}$. Then in $\bar{\sigma}_{\mid W}: \mathcal{O}_{n \mid W} \rightarrow \mathcal{O}_{n \mid W} \rightarrow \mathbb{C}^{m_{n}}$ does not vanish at any point. It follows that

$$
\operatorname{coker}\left(\bar{\sigma}_{\mid W}\right) \simeq\left(\bigoplus_{i=1}^{n-1} \mathcal{O}_{i \mid W} \otimes \mathbb{C}^{m_{i}}\right) \oplus\left(\mathcal{O}_{n \mid W} \otimes \mathbb{C}^{m_{n}-1}\right)
$$

From [13], exp. IV, cor. 5.7, $\mathcal{F}=\operatorname{coker}\left(\bar{\sigma}_{\mid T}\right)$ is flat on $X$. It is a family of quasi locally free sheaves of type $\left(m_{1}, \ldots, m_{n-1}, m_{n}-1\right)$, and it is easy to verify that it is a good family. From the induction hypothesis we can assume, by replacing $T$ with a smaller affine neighbourhood of $Q$, that

$$
\mathcal{F} \simeq\left(\bigoplus_{i=1}^{n-1} \mathcal{O}_{\left(X \times C_{i}\right) \cap T} \otimes \mathbb{C}^{m_{i}}\right) \oplus\left(\mathcal{O}_{T} \otimes \mathbb{C}^{m_{n}-1}\right)
$$


Hence we have an exact sequence

$$
0 \longrightarrow \mathcal{O}_{T} \longrightarrow \mathbb{E}_{\mid T} \longrightarrow\left(\bigoplus_{i=1}^{n-1} \mathcal{O}_{\left(X \times C_{i}\right) \cap T} \otimes \mathbb{C}^{m_{i}}\right) \oplus\left(\mathcal{O}_{T} \otimes \mathbb{C}^{m_{n}-1}\right) \longrightarrow 0
$$

Now we have $\operatorname{Ext}_{\mathcal{O}_{T}}^{1}\left(\mathcal{O}_{\left(X \times C_{i}\right) \cap T}, \mathcal{O}_{T}\right)=\{0\}$ for $1 \leq i \leq n$ : it suffices to prove that $\mathcal{E} x t_{\mathcal{O}_{T}}^{1}\left(\mathcal{O}_{\left(X \times C_{i}\right) \cap T}, \mathcal{O}_{T}\right)=0$. This follows easily from the resolution

$$
\cdots \longrightarrow \mathcal{O}_{T} \stackrel{\times \zeta^{i}}{\longrightarrow} \mathcal{O}_{T} \stackrel{\times \zeta^{n-i}}{\longrightarrow} \mathcal{O}_{T} \stackrel{\times \zeta^{i}}{\longrightarrow} \mathcal{O}_{T} \longrightarrow \mathcal{O}_{\left(X \times C_{i}\right) \cap T} .
$$

Hence

$$
\mathbb{E}_{\mid T} \simeq \bigoplus_{i=1}^{n} \mathcal{O}_{\left(X \times C_{i}\right) \cap T} \otimes \mathbb{C}^{m_{i}}
$$

and the result is proved for $m_{n}$.

Let $\mathbb{E}$ be a good family of quasi locally free sheaves of rigid type parametrised by $X$, and $x \in X$ a closed point. Suppose that $\mathbb{E}_{x}$ is simple. Let $\left(S, s_{0}, \mathcal{E}, \alpha\right)$ be a semi-universal deformation of $\mathbb{E}_{x}$ (cf. 2.2.1). Let $f: S(x) \rightarrow S$ be the morphism induced by $\mathbb{E}$ (where $S(x)$ is the germ defined by $\mathbb{E}$ around $x$ ). Then $T S_{s_{0}}$ is canonically isomorphic to $\operatorname{Ext}_{\mathcal{O}_{Y}}^{1}\left(\mathbb{E}_{x}, \mathbb{E}_{x}\right)$, and by [6], th. 6.10 and cor. 6.11, we have

$$
T S_{\text {reg, } s_{0}}=H^{1}\left(\mathcal{E} n d\left(\mathbb{E}_{x}\right)\right) .
$$

It follows easily from theorem 3.2.1 that the image of $T f_{x}: T X_{x} \rightarrow T S_{s_{0}}$ is contained in $T S_{\text {reg, } s_{0}}$ and that the image of $f$ is contained in $S_{\text {reg. }}$. Hence if $\boldsymbol{M}$ is the moduli space of stable sheaves corresponding to $\mathbb{E}_{x}$ and $X$ is connected, the image of the canonical morphism $f_{\mathbb{E}}: X \rightarrow M$ associated to $\mathbb{E}$ is contained in $\boldsymbol{M}_{\text {reg }}$.

\section{Coherent SheAves on Reducible Deformations of PRimitive Double CuRves}

\subsection{Maximal Reducible Deformations}

(cf. [9], [10], [11])

Let $C$ be a projective irreducible smooth curve and $Y=C_{2}$ a primitive double curve, with underlying smooth curve $C$, and associated line bundle $L$ on $C$. Let $S$ be a smooth curve, $P \in S$ and $\pi: \mathcal{C} \rightarrow S$ a maximal reducible deformation of $Y$ (cf. [9]). This means that

(i) $\mathcal{C}$ is a reduced algebraic variety with two irreducible components $\mathcal{C}_{1}, \mathcal{C}_{2}$.

(ii) We have $\pi^{-1}(P)=Y$. So we can view $C$ as a curve in $\mathcal{C}$.

(iii) For $i=1,2$, let $\pi_{i}: \mathcal{C}_{i} \rightarrow S$ be the restriction of $\pi$. Then $\pi_{i}^{-1}(P)=C$ and $\pi_{i}$ is a flat family of smooth irreducible projective curves.

(iv) For every $z \in S \backslash\{P\}$, the components $\mathcal{C}_{1, z}, \mathcal{C}_{2, z}$ of $\mathcal{C}_{z}$ meet transversally. 
For every $z \in S \backslash\{P\}, \mathcal{C}_{1, z}$ and $\mathcal{C}_{2, z}$ meet in exactly $-\operatorname{deg}(L)$ points. If $\operatorname{deg}(L)=0$, then $\pi$ (or $\mathcal{C})$ is called a fragmented deformation.

Let $z \subset \mathcal{C}$ be the closure in $\mathcal{C}$ of the locus of the intersection points of the components of $\pi^{-1}(z)$, $z \neq P$. Since $S$ is a curve, $z$ is a curve of $\mathcal{C}_{1}$ and $\mathcal{C}_{2}$. It intersects $C$ in a finite number of points. If $x \in C$, let $r_{x}$ be the number of branches of $z$ at $x$ and $s_{x}$ the sum of the multiplicities of the intersections of these branches with $C$. If $x \in z$, then the branches of $z$ at $x$ intersect transversally with $C$, and we have $r_{x}=s_{x}$. We have

$$
L \simeq \mathcal{O}_{C}\left(-\sum_{x \in z \cap C} r_{x} x\right) \simeq \mathcal{J}_{z \cap C, C}
$$

For every $\in C$, there exists an unique integer $p>0$ such that $\mathcal{J}_{C, x} /\left\langle\left(\pi_{1}, \pi_{2}\right)\right\rangle$ is generated by the image of $\left(\pi_{1}^{p} \lambda_{1}, 0\right)$, for some $\lambda_{1} \in \mathcal{O}_{\mathcal{C}_{1}, x}$ not divisible by $\pi_{1}$. Moreover $\left(\pi_{1}^{p} \lambda_{1}, 0\right)$ is a generator of the ideal $\mathcal{J}_{\mathcal{C}_{1}, \mathcal{C}, x}$ of $\mathcal{C}_{2}$ in $\mathcal{C}$, and $\lambda_{1}$ is a generator of the ideal of $z$ in $\mathcal{C}_{1}$ at $x$. The integer $p$ does not depend on $x$. Of course we have a symmetric result: $\mathcal{J}_{C, x} /\left\langle\left(\pi_{1}, \pi_{2}\right)\right\rangle$ is generated by the image of $\left(0, \pi_{2}^{p} \lambda_{2}\right)$, for some $\lambda_{2} \in \mathcal{O}_{\mathcal{C}_{2}, x}$ not divisible by $\pi_{2}$. Moreover $\left(0, \pi_{2}^{p} \lambda_{2}\right)$ is a generator of the ideal $\mathcal{J}_{\mathcal{C}_{2}, \mathcal{C}, x}$ of $\mathcal{C}_{1}$ in $\mathcal{C}$, and $\lambda_{2}$ is a generator of the ideal of $\mathcal{Z}$ in $\mathcal{C}_{2}$ at $x$. We can even assume that $\left(\lambda_{1}, \lambda_{2}\right) \in \mathcal{O}_{\mathcal{C}, x}$.

Let

$$
z_{0}=\mathcal{e}_{1} \cap \mathcal{C}_{2} \subset \mathcal{e}
$$

We have then $\left(Z_{0}\right)_{\text {red }}=\mathcal{Z} \cup C$ (and $Z_{0}=\mathcal{Z} \cup C$ if $p=1$ ). The ideal sheaf $\mathbb{L}_{1}=\mathcal{J}_{Z_{0}, e_{1}}$ (resp. $\mathbb{L}_{2}=\mathcal{J}_{Z_{0}, \mathfrak{C}_{2}}$ ) of $Z_{0}$ in $\mathcal{C}_{1}$ (resp. $\mathcal{C}_{2}$ ) at $x$ is generated by $\lambda_{1} \pi_{1}^{p}$ (resp. $\lambda_{2} \pi_{2}^{p}$ ). Hence $\mathbb{L}_{1}$ (resp. $\left.\mathbb{L}_{2}\right)$ is a line bundle on $\mathcal{C}_{1}$ (resp. $\left.\mathcal{C}_{2}\right)$. The ideal sheaf $\mathcal{J}_{Z, \mathfrak{C}_{1}}\left(\right.$ resp. $\left.\mathcal{J}_{Z, \mathfrak{C}_{2}}\right)$ of $z$ in $\mathcal{C}_{1}\left(\right.$ resp. $\left.\mathcal{C}_{2}\right)$ is canonically isomorphic to $\mathbb{L}_{1}$ (resp. $\mathbb{L}_{2}$ ). The $p$-th infinitesimal neighbourhoods of $C$ in $\mathcal{C}_{1}$, $\mathrm{C}_{2}$ (generated respectively by $\pi_{1}^{p}$ and $\pi_{2}^{p}$ ) are canonically isomorphic, we will denote them by $C^{(p)}$. We have also a canonical isomorphism $\mathbb{L}_{1 \mid C^{(p)}} \simeq \mathbb{L}_{2 \mid C^{(p)}}$, and $\mathbb{L}_{1 \mid C} \simeq \mathbb{L}_{2 \mid C} \simeq L$. It is also possible, by replacing $S$ with a smaller neighbourhood of $P$, to assume that $\mathbb{L}_{1 \mid z_{0}} \simeq \mathbb{L}_{2 \mid z_{0}}$. Let $\mathbb{L}=\mathbb{L}_{1 \mid z_{0}}=\mathbb{L}_{2 \mid z_{0}}$.

We have $\mathcal{J}_{\mathfrak{C}_{1}, \mathrm{e}}=\mathbb{L}_{2}$ and $\mathcal{J}_{\mathfrak{C}_{2}, \mathrm{e}}=\mathbb{L}_{1}$.

In this paper we will always assume that $p=1$.

There exists a maximal reducible deformation of $Y$ either if $\operatorname{deg}(L)=0$, or if $\operatorname{deg}(L)<0$ and there exists $-\operatorname{deg}(L)$ distinct points $P_{1}, \ldots, P_{d}$ of $C$ (with $d=-\operatorname{deg}(L)$ ) such that $L=\mathcal{O}_{C}\left(-P_{1}-\cdots-P_{d}\right)$. And in the second case we can even assume that

$z \cap C=\left\{P_{1}, \ldots, P_{d}\right\}$.

\subsection{Coherent Sheaves on Reduced Reducible Curves}

(cf. [11], 4-)

Let $D$ be a projective curve with two components $D_{1}, D_{2}$ intersecting transversally, and $Z=D_{1} \cap D_{2}$. Let $\mathcal{E}$ be a coherent sheaf on $D$. Then the following conditions are equivalent:

(i) $\mathcal{E}$ is pure of dimension 1 .

(ii) $\mathcal{E}$ is of depth 1 . 
(iii) $\mathcal{E}$ is locally free at every point of $X$ belonging to only one component, and if $x \in Z$, then there exist integers $a, a_{1}, a_{2} \geq 0$ and an isomorphism

$$
\mathcal{E}_{x} \simeq a \mathcal{O}_{X, x} \oplus a_{1} \mathcal{O}_{D_{1}, x} \oplus a_{2} \mathcal{O}_{D_{2}, x}
$$

(iv) $\mathcal{E}$ is torsion free, i.e. for every $x \in X$, every element of $\mathcal{O}_{X, x}$ which is not a zero divisor in $\mathcal{O}_{X, x}$ is not a zero divisor in $\mathcal{E}_{x}$.

(v) $\mathcal{E}$ is reflexive.

Let $E_{i}=\mathcal{E}_{\mid D_{i}} / T_{i}$, where $T_{i}$ is the torsion subsheaf. It is a vector bundle on $D_{i}$. Let $x \in Z$. Then there exists a finite dimensional vector space $W$, surjective maps $f_{i}: E_{i, x} \rightarrow W$, such that the $\mathcal{O}_{D, x}$-module $\mathcal{E}_{x}$ is isomorphic to $\left\{\left(\phi_{1}, \phi_{2}\right) \in E_{1}(x) \times E_{2}(x) ; f_{1}\left(\phi_{1}(x)\right)=f_{2}\left(\phi_{2}(x)\right)\right\}$ (where $E_{i}(x)$ is the fibre at $x$ of the sheaf $E_{i}$, and $E_{i, x}$ the fibre of the corresponding vector bundle). We have then

$$
\mathcal{E}_{x} \simeq\left(W \times \mathcal{O}_{D, x}\right) \oplus\left(\operatorname{ker}\left(f_{1}\right) \otimes \mathcal{O}_{D_{1}, x}\right) \oplus\left(\operatorname{ker}\left(f_{2}\right) \otimes \mathcal{O}_{D_{2}, x}\right) .
$$

We say that the sheaf is linked at $x$ if $W$ has the maximal possible dimension, i.e. $\operatorname{dim}(W)=\inf \left(r k\left(E_{1}\right), \operatorname{rk}\left(E_{2}\right)\right)$ (i.e. if in (iii) $a_{1}=0$ or $a_{2}=0$ ). We say that $\mathcal{E}$ is linked if it is linked at every point of $Z$.

\subsection{Regular Sheaves}

A coherent sheaf $\mathcal{E}$ on $\mathcal{C}$ is called regular if it is locally free on $\mathcal{C} \backslash z_{0}$, and if for every $x \in z_{0}$ there exists a neighbourhood of $x$ in $\mathcal{C}$, a vector bundle $\mathbb{E}$ on $U, i \in\{1,2\}$, and a vector bundle $F$ on $U \cap \mathcal{C}_{i}$, such that $\mathcal{E}_{\mid U} \simeq \mathbb{E} \oplus F$.

Let $\mathcal{E}$ be a coherent sheaf on $\mathcal{C}$. Then by [11], prop. 6.4.3, the following assertions are equivalent:

(i) $\mathcal{E}$ is regular (with $i=1$ ).

(ii) There exists an exact sequence $0 \rightarrow E_{2} \rightarrow \mathcal{E} \rightarrow E_{1} \rightarrow 0$, where for $j=1,2, E_{j}$ is a vector bundle on $\mathcal{C}_{j}$, such that the associated morphism $E_{1 \mid z_{0}} \rightarrow \mathbb{L}^{*} \otimes E_{2 \mid z_{0}}$ is surjective on a neighbourhood of $C$.

(iii) There exists an exact sequence $0 \rightarrow E_{1} \rightarrow \mathcal{E} \rightarrow E_{2} \rightarrow 0$, where for $j=1,2, E_{j}$ is a vector bundle on $\mathcal{C}_{j}$, such that the associated morphism $E_{2 \mid z_{0}} \rightarrow \mathbb{L}^{*} \otimes E_{1 \mid z_{0}}$ is injective (as a morphism of vector bundles) on a neighbourhood of $C$.

If we restrict the exact sequence of (ii) to $Y$ we get the canonical one

$$
0 \longrightarrow\left(\mathcal{E}_{\mid Y}\right)_{1} \longrightarrow \mathcal{E}_{\mid Y} \longrightarrow\left(\mathcal{E}_{\mid Y}\right)_{\mid C} \longrightarrow 0
$$

(cf. 2.1) and if we restrict the exact sequence of (iii) to $Y$ we get

$$
0 \longrightarrow\left(\mathcal{E}_{\mid Y}\right)^{(1)} \longrightarrow \mathcal{E}_{\mid Y} \longrightarrow\left(\mathcal{E}_{\mid Y}\right)^{(2)}=\left(\mathcal{E}_{\mid Y}\right)_{1} \otimes L^{*} \longrightarrow 0
$$

In particular $\mathcal{E}_{\mid Y}$ is quasi locally free, and for $s \in \backslash\{P\}$ in a neighbourhood of $P$, $\mathcal{E}_{s}$ is a linked torsion free sheaf.

We have a similar result by taking $i=2$.

For example, let $\mathcal{E}$ be a coherent sheaf on $\mathcal{C}$, flat on $S$. Suppose that for every $s \in S, \mathcal{E}_{s}$ is torsion free, and that $\mathcal{E}_{\mid Y}$ is quasi locally free of rigid type (cf. [3). Then $\mathcal{E}$ is regular ([11], prop. 6.4.5). 


\section{KODAÏRA-SPENCER ELEMENTS}

We keep the notations of 4.1 .

\subsection{Self-extensions of $\mathcal{O}_{C, x}$ ON $Y$}

We will need in 5.2 a description of the extensions

$$
0 \longrightarrow \mathcal{O}_{C, x} \longrightarrow \mathcal{E} \longrightarrow \mathcal{O}_{X, x} \longrightarrow 0
$$

on $Y$.

Let $x \in C$. Let $z \in \mathcal{O}_{Y, x}$ be an equation of $C$ and $t \in \mathcal{O}_{Y, x}$ over a generator of the maximal ideal of $\mathcal{O}_{C, x}$. The extensions (11) are parametrised by $\operatorname{Ext}_{\mathcal{O}_{Y, x}}^{1}\left(\mathcal{O}_{C, x}, \mathcal{O}_{C, x}\right)$, which is isomorphic to $\mathcal{O}_{C, x}$. This can be seen easily by using the free resolution of $\mathcal{O}_{C, x}$ on $Y$ :

$$
\cdots \longrightarrow \mathcal{O}_{Y, x} \stackrel{\times z}{\longrightarrow} \mathcal{O}_{Y, x} \stackrel{\times z}{\longrightarrow} \mathcal{O}_{C, x} \longrightarrow 0
$$

For every positive integer $n$, let

$$
\mathcal{J}_{Y, n}=\left(z, t^{n}\right) \subset \mathcal{O}_{Y, x}, \quad \mathcal{J}_{C, n}=\left(t^{n}\right) \subset \mathcal{O}_{C, x}
$$

(the ideals of $n x$ ). Then we have an obvious extension

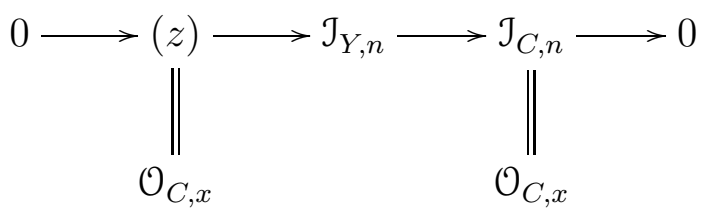

and it is easy to see that it is associated to $t^{n} \in \operatorname{Ext}_{\mathcal{O}_{Y, x}}^{1}\left(\mathcal{O}_{C, x}, \mathcal{O}_{C, x}\right)$.

\subsection{Proof of the MAIN RESUlT}

Let $\mathcal{E}^{[1]}, \mathcal{E}^{[2]}$ be coherent sheaves on $\mathcal{C}$, flat on $S$. Suppose that $\mathcal{E}_{\mid Y}^{[1]}, \mathcal{E}_{\mid Y}^{[2]}$ are isomorphic. Let

$$
E=\mathcal{E}_{\mid Y}^{[1]}=\mathcal{E}_{\mid Y}^{[2]}
$$

Suppose that $E$ is quasi locally free of rigid type, and that for every $s \in S, \mathcal{E}_{s}^{[1]}$ and $\mathcal{E}_{s}^{[2]}$ are torsion free. Then $\mathcal{E}^{[1]}$ and $\mathcal{E}^{[2]}$ are regular (cf. 4.3). Suppose that for every $s \in S \backslash\{P\}, \mathcal{E}_{s}^{[1]}$ and $\mathcal{E}_{s}^{[2]}$ are linked (this is always true on a neighbourhood of $P$ ). It follows that there exists an integer $r$ such that for $i=1,2$, for every $s \in S \backslash\{P\}, \mathcal{E}_{s}^{[i]}$ is of rank $r$ on $\mathcal{C}_{1, s} \backslash Z$ and $r+1$ on $\mathcal{C}_{2, s} \backslash \mathcal{Z}$, or of rank $r$ on $\mathcal{C}_{2, s} \backslash \mathcal{Z}$ and $r+1$ on $\mathcal{C}_{1, s} \backslash \mathcal{Z}$. We suppose that $r>0$. We will consider two cases:

\section{Case A}

$-\mathcal{E}_{s}^{[1]}$ is of rank $r$ on $\mathcal{C}_{2, s} \backslash \mathcal{Z}$ and of rank $r+1$ on $\mathcal{C}_{1, s} \backslash \mathcal{Z}$.

$-\mathcal{E}_{s}^{[2]}$ is of rank $r+1$ on $\mathcal{C}_{2, s} \backslash \mathcal{Z}$ and of rank $r$ on $\mathcal{C}_{1, s} \backslash \mathcal{Z}$.

\section{Case B}

$-\mathcal{E}_{s}^{[1]}$ and $\mathcal{E}_{s}^{[2]}$ are of rank $r$ on $\mathcal{C}_{2, s} \backslash \mathcal{Z}$ and of rank $r+1$ on $\mathcal{C}_{1, s} \backslash \mathcal{Z}$. 
We want to study $\omega_{\mathcal{E}[1], \mathcal{E}[2]} \in \operatorname{Ext}_{\mathcal{O}_{Y}}^{1}(E, E)$ (cf. [2.3) .

Recall that $\mathcal{E} x t_{\mathcal{O}_{Y}}^{1}(E, E) \simeq H^{0}\left(L^{*}\right)$ (cf. 3.1.2, 3.1.3). From 4.1, $\mathcal{C}$ induces a one dimensional subspace $\Delta \subset H^{0}\left(L^{*}\right)$.

Let $Y_{2}$ be the second infinitesimal neighbourhood of $Y$ in $\mathcal{C}$. Let $t \in \mathcal{O}_{S, P}$ be a generator of the maximal ideal. We will also denote by $\pi$ (resp. $\left.\pi_{i}, i=1,2\right)$ the regular function $t \circ \pi$ (resp. $t \circ \pi_{i}$ ) defined on a neighbourhood of $C$. Then $Y_{2}$ is defined in a neighbourhood of $Y$ by the equation $\pi^{2}=0$. Let $\mathcal{J}$ be the ideal sheaf of $Y$ in $Y_{2}$. We have $\mathcal{J} \simeq \mathcal{O}_{Y}$. For $i=1,2$ we have a canonical exact sequence

$$
0 \longrightarrow E \otimes \mathcal{J} \simeq E \longrightarrow \mathcal{E}_{\mid Y_{2}}^{[i]} \longrightarrow E \longrightarrow 0
$$

associated to $\sigma_{i} \in \operatorname{Ext}_{\mathcal{O}_{Y_{2}}}^{1}(E \otimes \mathcal{J}, E)$.

Given an extension $0 \rightarrow E \otimes \mathcal{J} \rightarrow \mathcal{F} \rightarrow E \rightarrow 0$ on $Y_{2}$, the canonical morphism $\mathcal{F} \otimes \mathcal{J} \rightarrow \mathcal{F}$ induces an endomorphism of $E$. In this way we get a canonical morphism

$\operatorname{Ext}_{\mathcal{O}_{Y_{2}}}^{1}(E, E \otimes \mathcal{J}) \rightarrow \operatorname{End}(E)$, whose kernel corresponds to extensions such that $\mathcal{F}$ is concentrated on $Y$. Hence we have an exact sequence

$$
0 \longrightarrow \operatorname{Ext}_{\mathcal{O}_{Y}}^{1}(E \otimes \mathcal{J}, E) \longrightarrow \operatorname{Ext}_{\mathcal{O}_{Y_{2}}}^{1}(E \otimes \mathcal{J}, E) \stackrel{\theta}{\longrightarrow} \operatorname{End}(E)
$$

The image of $\sigma_{i}, i=1,2$, is $I_{E}$. Hence, by using the action of $\operatorname{Aut}(E)$, we see that $\theta$ is surjective, and that we have an exact sequence

$$
0 \longrightarrow \operatorname{Ext}_{\mathcal{O}_{Y}}^{1}(E \otimes \mathcal{J}, E) \longrightarrow \operatorname{Ext}_{\mathcal{O}_{Y_{2}}}^{1}(E \otimes \mathcal{J}, E) \stackrel{\theta}{\longrightarrow} \operatorname{End}(E) \longrightarrow 0
$$

Recall that

Let

$$
\omega_{\mathcal{E}^{[1], \mathcal{E}}[2]}=\sigma_{1}-\sigma_{2} .
$$

$$
\phi: \operatorname{Ext}_{\mathcal{O}_{Y}}^{1}(E, E) \longrightarrow H^{0}\left(\mathcal{E} x t_{\mathcal{O}_{Y}}^{1}(E, E)\right)
$$

be the canonical morphism.

5.2.1. Theorem : $\mathbf{1}-$ In case $A, \phi\left(\omega_{\mathcal{E}^{[1], \varepsilon^{[2]}}}\right)$ generates $\Delta$.

$\mathbf{2}-$ in case $B$, we have $\phi\left(\omega_{\mathcal{E}^{[1]}, \mathcal{E}^{[2]}}\right)=0$.

Proof. We will only prove $\mathbf{1}$. The proof of $\mathbf{2}$ follows easily.

Let $x \in C$. Since $E_{x} \simeq r \mathcal{O}_{Y, x} \oplus \mathcal{O}_{C, x}$, we have $\operatorname{Ext}_{\mathcal{O}_{Y, x}}^{1}\left(E_{x}, E_{x}\right) \simeq \operatorname{Ext}_{\mathcal{O}_{Y}, x}^{1}\left(\mathcal{O}_{C, x}, \mathcal{O}_{C, x}\right)$. We will give an explicit description of the extension

$$
0 \longrightarrow \mathcal{O}_{C, x} \longrightarrow \mathcal{V} \longrightarrow \mathcal{O}_{C, x} \longrightarrow 0
$$

corresponding to $\phi\left(\omega_{\mathcal{E}}^{[1], \mathcal{E}}[2]\right)(x) \in \operatorname{Ext}_{\mathcal{O}_{Y}}^{1}\left(\mathcal{O}_{C, x}, \mathcal{O}_{C, x}\right)$, and from [5.1, 1 will follow from the fact that $\mathcal{V} \simeq \mathcal{O}_{Y, x}$ if $x \notin Z \cap C$, and $\mathcal{V} \simeq \mathcal{J}_{Y, 1}$ if $x \in \mathcal{Z} \cap C$.

Let $\tau_{1}, \tau_{2} \in \operatorname{Ext}_{\mathcal{O}_{Y_{2}, x}}^{1}\left(E_{x}, E_{x}\right)$ be the images of $\sigma_{1}, \sigma_{2}$ respectively. We have also an exact sequence

$$
0 \longrightarrow \operatorname{Ext}_{\mathcal{O}_{Y, x}}^{1}\left(E_{x}, E_{x}\right) \longrightarrow \operatorname{Ext}_{\mathcal{O}_{Y_{2}, x}}^{1}\left(E_{x}, E_{x}\right) \longrightarrow \operatorname{End}\left(E_{x}\right)
$$


and $\tau_{1}-\tau_{2} \in \operatorname{Ext}_{\mathcal{O}_{Y, x}}^{1}\left(E_{x}, E_{x}\right)$. In a neighbourhood of $x$ in $\mathcal{C}, \mathcal{E}^{[1]}$ is isomorphic to $r \mathcal{O}_{\mathcal{C}} \oplus \mathcal{O}_{\mathcal{C}_{1}}$, and $\mathcal{E}^{[2]}$ is isomorphic to $r \mathcal{O}_{\mathcal{C}} \oplus \mathcal{O}_{\mathcal{C}_{2}}$. We can suppose that these isomorphisms are the same on $Y$. The exact sequence (2) is the canonical exact sequence

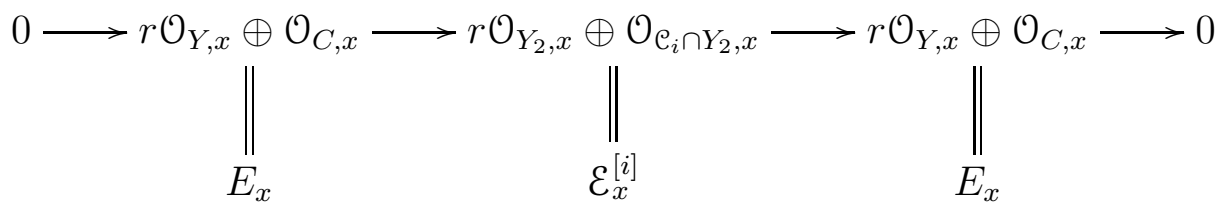

Note that $\mathcal{O}_{\mathfrak{C}_{i} \cap Y_{2}, x}=\mathcal{O}_{\mathfrak{C}_{i}, x} /\left(\pi_{1}^{2}\right)$. We have

$$
\tau_{1}-\tau_{2} \in \operatorname{Ext}_{\mathcal{O}_{Y_{2}, x}}^{1}\left(\mathcal{O}_{C, x}, \mathcal{O}_{C, x}\right) \subset \operatorname{Ext}_{\mathcal{O}_{Y_{2}, x}}^{1}\left(E_{x}, E_{x}\right)
$$

We have $\tau_{1}-\tau_{2}=\eta_{1}-\eta_{2}$, where $\eta_{i} \in \operatorname{Ext}_{\mathcal{O}_{Y_{2}, x}}^{1}\left(\mathcal{O}_{C, x}, \mathcal{O}_{C, x}\right)$ is associated to the canonical exact sequence

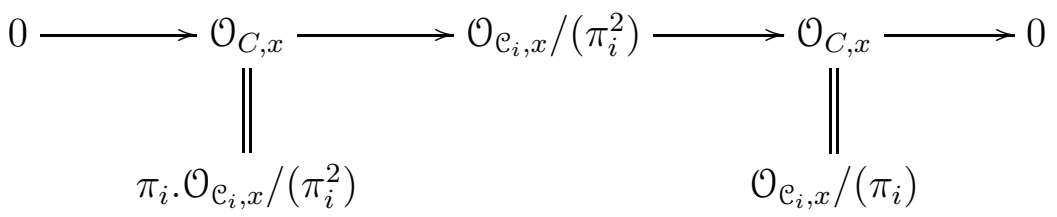

If $\beta \in \mathcal{O}_{\mathfrak{C}_{i}, x}$, the image of $\pi_{i} \beta$ in $\mathcal{O}_{\mathfrak{C}_{i}, x} /\left(\pi_{i}^{2}\right)$ depends only on $\beta_{\mid C}$. So for every $\alpha \in \mathcal{O}_{C, x}$ we can define $\pi_{i} \alpha \in \mathcal{O}_{\mathcal{C}_{i}, x} /\left(\pi_{i}^{2}\right)$. Let

$$
\mathcal{N}=\left\{\left(\pi_{1} \alpha,-\pi_{2} \alpha\right) \in \mathcal{O}_{\mathcal{C}_{i}, x} /\left(\pi_{1}^{2}\right) \times \mathcal{O}_{\mathfrak{C}_{2}, x} /\left(\pi_{2}^{2}\right) ; \alpha \in \mathcal{O}_{C, x}\right\},
$$

which is a sub- $\mathcal{O}_{\mathcal{C}, x}$-module of $\mathcal{O}_{\mathfrak{C}_{i}, x} /\left(\pi_{1}^{2}\right) \times \mathcal{O}_{\mathfrak{C}_{2}, x} /\left(\pi_{2}^{2}\right)$. Let

$$
\mathcal{U}=\left[\mathcal{O}_{\mathfrak{e}_{i}, x} /\left(\pi_{1}^{2}\right) \times \mathcal{O}_{\mathfrak{C}_{2}, x} /\left(\pi_{2}^{2}\right)\right] / \mathcal{N} \text {. }
$$

The morphism

$$
\begin{gathered}
\Phi: \mathcal{U} \longrightarrow \mathcal{O}_{C, x} \times \mathcal{O}_{C, x} \\
\left(\alpha_{1}, \alpha_{2}\right) \longmapsto\left(\alpha_{1 \mid C}, \alpha_{2 \mid C}\right)
\end{gathered}
$$

is surjective. We have

$$
\operatorname{ker}(\Phi)=\left\{\left(\pi_{1} \lambda_{1}, \pi_{2} \lambda_{2}\right) ; \lambda_{1}, \lambda_{2} \in \mathcal{O}_{C, x}\right\} / \mathcal{N} .
$$

We have $\operatorname{ker}(\Phi) \simeq \mathcal{O}_{C, x}$, the isomorphism being defined by

$$
\begin{aligned}
\nu: & \mathcal{O}_{C, x} \longrightarrow \operatorname{ker}(\Phi) \\
& \alpha \longmapsto\left(\pi_{1} \alpha, 0\right)=\left(0, \pi_{2} \alpha\right) .
\end{aligned}
$$

Hence we have an exact sequence

$$
0 \longrightarrow \mathcal{O}_{C, x} \longrightarrow \mathcal{U} \longrightarrow \mathcal{O}_{C, x} \oplus \mathcal{O}_{C, x} \longrightarrow 0 \text {. }
$$

We have an inclusion

$$
\begin{aligned}
\mu_{1}: \mathcal{O}_{\mathfrak{C}_{1}, x} /\left(\pi_{1}^{2}\right) & \longrightarrow \mathcal{U} \\
\alpha_{1} & \longmapsto\left(\alpha_{1}, 0\right),
\end{aligned}
$$


and similarly $\mu_{2}: \mathcal{O}_{\mathcal{C}_{2}, x} /\left(\pi_{2}^{2}\right) \hookrightarrow \mathcal{U}$. We have a commutative diagram with exact rows

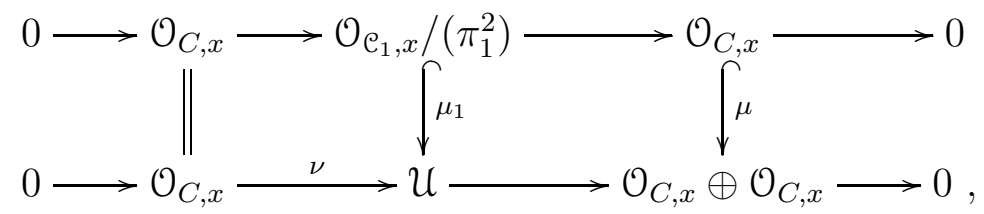

where $\mu$ is the inclusion in the first factor.

Let $\gamma \in \operatorname{Ext}_{Y_{2}, x}^{1}\left(\mathcal{O}_{C, x} \oplus \mathcal{O}_{C, x}, \mathcal{O}_{C, x}\right)=\operatorname{Ext}_{Y_{2}, x}^{1}\left(\mathcal{O}_{C, x}, \mathcal{O}_{C, x}\right) \oplus \operatorname{Ext}_{Y_{2}, x}^{1}\left(\mathcal{O}_{C, x}, \mathcal{O}_{C, x}\right) \quad$ associated to (3). From the preceding diagram and prop. 4.3 .1 of [3], the first component of $\gamma$ is $\eta_{1}$. Similarly the second component of $\gamma$ is $\eta_{2}$. So we have $\gamma=\left(\eta_{1}, \eta_{2}\right)$. It follows that $\eta_{1}-\eta_{2}$ corresponds to the top exact sequence in the following commutative diagram

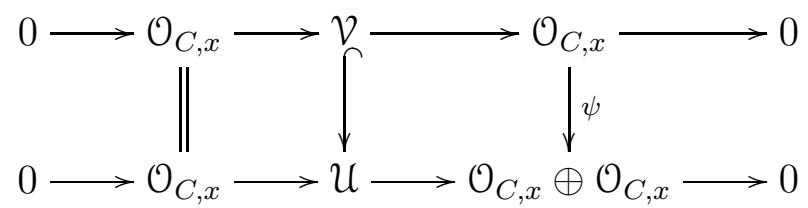

where $\psi: \alpha \mapsto(\alpha,-\alpha)$, and

$$
\mathcal{V}=\left\{(\alpha, \beta) \in \mathcal{U} ; \alpha_{\mid C}+\beta_{\mid C}=0\right\} .
$$

If $\left(u_{1}, u_{2}\right) \in \mathcal{O}_{\mathfrak{C}_{i}, x} /\left(\pi_{1}^{2}\right) \times \mathcal{O}_{\mathfrak{C}_{2}, x} /\left(\pi_{2}^{2}\right)$ is such that $u_{1 \mid C}+u_{2 \mid C}=0$, we will denote by $\left[u_{1}, u_{2}\right]$ the corresponding element of $\mathcal{V}$.

We have $\pi \mathcal{V}=\{0\}$, i.e. the top exact sequence is a sequence of $\mathcal{O}_{Y, x^{-}}$modules.

The case $x \notin z \cap C-$ We have then

$$
\mathcal{O}_{\mathfrak{C}, x}=\left\{\left(\alpha_{1}, \alpha_{2}\right) \in \mathcal{O}_{\mathfrak{C}_{1}, x} \times \mathcal{O}_{\mathfrak{C}_{2}, x} ; \alpha_{1 \mid C}=\alpha_{2 \mid C}\right\} .
$$

Let

$$
\begin{aligned}
\mathbf{f}: \mathcal{O}_{\mathcal{C}, x} \longrightarrow \mathcal{V} \\
1 \longmapsto[1,-1] .
\end{aligned}
$$

We now prove that $\mathbf{f}$ induces an isomorphism $\mathcal{O}_{Y, x} \simeq \mathcal{V}$. It is obvious that $\mathbf{f}$ is surjective and that $(\pi) \subset \operatorname{ker}(\mathbf{f})$. Suppose that $\left(\alpha_{1}, \alpha_{2}\right) \in \mathcal{O}_{\mathcal{C}, x}$ is such that $\mathbf{f}\left(\alpha_{1}, \alpha_{2}\right)=0$. We can then write

$$
\left(\alpha_{1},-\alpha_{2}\right)=\left(\pi_{1} \beta_{1},-\pi_{2} \beta_{2}\right)+\left(\pi_{1}^{2} \epsilon_{1},-\pi_{2} \epsilon_{2}^{2}\right),
$$

with $\beta_{1 \mid C}=\beta_{2 \mid C}$. Hence $\left(\beta_{1}, \beta_{2}\right) \in \mathcal{O}_{\mathcal{C}, x}$, and

$$
\left(\alpha_{1}, \alpha_{2}\right)=\pi \cdot\left[\left(\beta_{1}, \beta_{2}\right)+\left(\pi_{1} \epsilon_{1}, \pi_{2} \epsilon_{2}\right)\right] .
$$

We have $\left(\beta_{1}, \beta_{2}\right)+\left(\pi_{1} \epsilon_{1}, \pi_{2} \epsilon_{2}\right) \in \mathcal{O}_{\mathcal{C}_{x}}$, hence $\left(\alpha_{1}, \alpha_{2}\right) \in(\pi)$.

The case $x \in \mathcal{Z} \cap C$ - We have then an isomorphism

$$
\theta: \mathcal{O}_{\mathfrak{C}_{1}, x} /\left(\pi_{1} \lambda_{1}\right) \longrightarrow \mathcal{O}_{\mathfrak{C}_{2}, x} /\left(\pi_{2} \lambda_{2}\right)
$$

such that $\theta\left(\pi_{1}\right)=\pi_{2}, \theta(\alpha)_{\mid C}=\alpha_{\mid C}$ for every $\alpha \in \mathcal{O}_{\mathfrak{C}_{1}, x} /\left(\pi_{1} \lambda_{1}\right)$ (cf. 4.1). The restrictions $\lambda_{i \mid C}$, $i=1,2$ are generators of the maximal ideal of $\mathcal{O}_{C, x}$. We can also assume that $\theta\left(\lambda_{1}\right)=\lambda_{2}$. We have then

$$
\mathcal{O}_{\mathcal{C}, x}=\left\{\left(\alpha_{1}, \alpha_{2}\right) \in \mathcal{O}_{\mathcal{C}_{1}, x} \times \mathcal{O}_{\mathcal{C}_{2}, x} ; \theta\left(\alpha_{1}\right)=\alpha_{2}\right\}
$$


We now prove that $\mathcal{V} \simeq \mathcal{J}_{x}$ (the ideal sheaf of $\{x\}$ in $\left.Y\right)$. Let $z=\left(\pi_{1} \lambda_{1}, 0\right), t=\left(\lambda_{1}, \lambda_{2}\right)$ in $\mathcal{O}_{\mathcal{e}, x}$ (cf. (44)). We have $\mathcal{J}_{x}=(t, z), z$ is an equation of $C$ (in $Y$ ) and $t_{\mid C}$ is a generator of the maximal ideal of $\mathcal{O}_{C, x}$. Then there exists a unique morphism $\rho: \mathcal{J}_{x} \rightarrow \mathcal{V}$ such that

$$
\rho(t)=[1,-1], \quad \rho(z)=\left[\pi_{1}, 0\right] .
$$

To prove this we have only to show that if $\alpha, \beta \in \mathcal{O}_{Y, x}$ are such that $\alpha z+\beta t=0$, then we have $\beta[1,-1]+\alpha\left[\pi_{1}, 0\right]=0$ in $\mathcal{V}$. We have $\alpha z+\beta t=0$ if and only if we can write

$$
\alpha=\epsilon t+\gamma z, \quad \beta=-\epsilon z,
$$

with $\epsilon, \gamma \in \mathcal{O}_{Y, x}$. We have then

$$
\begin{aligned}
\beta[1,-1]+\alpha\left[\pi_{1}, 0\right] & =-\epsilon z[1,-1]+(\epsilon t+\gamma z)\left[\pi_{1}, 0\right] \\
& =\epsilon\left(\lambda_{1}, \lambda_{2}\right)\left[\pi_{1}, 0\right]-\epsilon\left(\pi_{1} \lambda_{1}, 0\right)[1,-1]+\gamma\left[\pi_{1}^{2} \lambda_{1}, 0\right] \\
& =0 .
\end{aligned}
$$

Now we show that $\rho$ is injective. Suppose that $\left(\alpha_{1}, \alpha_{2}\right),\left(\beta_{1}, \beta_{2}\right) \in \mathcal{O}_{\mathcal{C}, x}$ are such that

$$
\rho\left(\left(\alpha_{1}, \alpha_{2}\right) z+\left(\beta_{1}, \beta_{2}\right) t\right)=0 .
$$

Then we have

$$
\left(\alpha_{1}, \alpha_{2}\right)\left[\pi_{1}, 0\right]+\left(\beta_{1}, \beta_{2}\right)[1,-1]=\left[\alpha_{1} \pi_{1}+\beta_{1},-\beta_{2}\right]=0 .
$$

Hence we can write

$$
\left(\alpha_{1} \pi_{1}+\beta_{1},-\beta_{2}\right)=\left(\pi_{1} \tau_{1},-\pi_{2} \tau_{2}\right)+\left(\pi_{1}^{2} \theta_{1}, \pi_{2}^{2} \theta_{2}\right)
$$

for some $\tau_{i}, \theta_{i} \in \mathcal{O}_{\mathfrak{C}_{i}, x}$ such that $\tau_{1 \mid C}=\tau_{2 \mid C}$, i.e.

$$
\alpha_{1} \pi_{1}+\beta_{1}=\pi_{1} \tau_{1}+\pi_{1}^{2} \theta_{1}, \quad \beta_{2}=\pi_{2} \tau_{2}-\pi_{2}^{2} \theta_{2} .
$$

Let

$$
u=\left(\alpha_{1}, \alpha_{2}\right) z+\left(\beta_{1}, \beta_{2}\right) t=\left(\lambda_{1}\left(\alpha_{1} \pi_{1}+\beta_{1}\right), \lambda_{2} \beta_{2}\right) .
$$

From (5) we see that $\beta_{1}$ is a multiple of $\pi_{1}$, and $\beta_{2}$ a multiple of $\pi_{2}: \beta_{1}=\pi_{1} \beta_{1}^{\prime}, \beta_{2}=\pi_{2} \beta_{2}^{\prime}$. We have then

We have

$$
u=\left(\pi_{1} \lambda_{1}\left(\alpha_{1}+\beta_{1}^{\prime}\right), \pi_{2} \lambda_{2} \beta_{2}^{\prime}\right)
$$

$$
\alpha_{1}+\beta_{1}^{\prime}=\tau_{1}+\pi_{1} \theta_{1}, \beta_{2}^{\prime}=\tau_{2}-\pi_{2} \theta_{2} .
$$

Hence $\left(\alpha_{1}+\beta_{1}^{\prime}\right)_{\mid C}=\beta_{2 \mid C}^{\prime}$ and $\left(\lambda_{1}\left(\alpha_{1}+\beta_{1}^{\prime}\right), \lambda_{2} \beta_{2}^{\prime}\right) \in \mathcal{O}_{\mathfrak{e}, x}$. It follows that $u=0$ in $\mathcal{O}_{Y, x}$.

Now we show that $\rho$ is surjective. Let $[\alpha, \beta] \in \mathcal{V}$. Then $\alpha_{\mid C}=-\beta_{\mid C}$. Let $\mu \in \mathcal{O}_{\mathfrak{C}_{1}, x}$ be such that $(\mu,-\beta) \in \mathcal{O}_{\mathfrak{C}, x}$. We have

$$
[\alpha, \beta]-(\mu,-\beta)[1,-1]=[\alpha-\mu, 0] .
$$

We can write $\alpha-\mu=\pi_{1} \zeta, \zeta \in \mathcal{O}_{\mathfrak{C}_{1}, x}$. Let $\delta \in \mathcal{O}_{\mathcal{C}_{2}, x}$ be such that $(\zeta, \delta) \in \mathcal{O}_{\mathcal{C}, x}$. We have then

$$
[\alpha, \beta]=(\mu,-\beta)[1,-1]+(\zeta, \delta)\left[\pi_{1}, 0\right]=\rho((\mu,-\beta) t+(\zeta, \delta) z) \text {. }
$$




\section{REFERENCES}

[1] Bănică, C., Forster, O. Multiple structures on space curves. In: Sundararaman, D. (Ed.) Proc. of Lefschetz Centennial Conf. (10-14 Dec. Mexico), Contemporary Mathematics 58, AMS, 1986, 47-64.

[2] Bayer, D., Eisenbud, D. Ribbons and their canonical embeddings. Trans. of the Amer. Math. Soc., 1995, 347-3, 719-756.

[3] Drézet, J.-M. Déformations des extensions larges de faisceaux. Pacific Journ. of Math. 220, 2 (2005), 201297.

[4] Drézet, J.-M. Faisceaux cohérents sur les courbes multiples . Collect. Math. 2006, 57-2, 121-171.

[5] Drézet, J.-M. Paramétrisation des courbes multiples primitives Adv. in Geom. 2007, 7, 559-612.

[6] Drézet, J.-M. Faisceaux sans torsion et faisceaux quasi localement libres sur les courbes multiples primitives. Mathematische Nachrichten, 2009, 282-7, 919-952.

[7] Drézet, J.-M. Sur les conditions d'existence des faisceaux semi-stables sur les courbes multiples primitives. Pacific Journ. of Math. 2011, 249-2, 291-319.

[8] Drézet, J.-M. Courbes multiples primitives et déformations de courbes lisses. Annales de la Faculté des Sciences de Toulouse 22, 1 (2013), 133-154.

[9] Drézet, J.-M. Fragmented deformations of primitive multiple curves. Central European Journal of Mathematics 11, no 12 (2013), 2106-2137.

[10] Drézet, J.-M. Reducible deformations and smoothing of primitive multiple curves. Manuscripta Mathematica 148 (2015), 447-469.

[11] Drézet, J.-M. Reachable sheaves on ribbons and deformations of moduli spaces of sheaves. Preprint (2016).

[12] Eisenbud, D., Green, M. Clifford indices of ribbons. Trans. of the Amer. Math. Soc., 1995, 347-3, 757-765. scientifiques et industrielles 1252, Hermann, Paris (1964).

[13] Grothendieck, A. et al. SGA1. Revêtements Etales et Groupe Fondamental. SGA1. Lect. Notes in Math. 224. Springer-Verlag (1971).

[14] Hartshorne, R. Algebraic geometry. Grad. Texts in Math., Vol. 52, Springer (1977).

[15] Maruyama, M. Moduli of stable sheaves II. J. Math. Kyoto Univ. 18 (1978), 577-614.

[16] Simpson, C.T. Moduli of representations of the fundamental group of a smooth projective variety I. Publ. Math. IHES 79 (1994), 47-129.

[17] Siu Y., Trautmann, G. Deformations of coherent analytic sheaves with compact supports. Memoirs of the Amer. Math. Soc., Vol. 29, N. 238 (1981).

[18] Strømme, S.A. Deforming vector bundles on the projective plane. Math. Ann. 263 (1983), 385-397.

Institut de Mathématiques de Jussieu - Paris Rive Gauche, Case 247, 4 place Jussieu, F-75252 PARIS, France

E-mail address: jean-marc.drezet@imj-prg.fr 